\title{
A Comparison of the Characteristics of Planar and Axisymmetric Bluff-Body Combustors Operated under Stratified Inlet Mixture Conditions
}

\author{
G. Paterakis, K. Souflas, E. Dogkas, and P. Koutmos \\ Laboratory of Applied Thermodynamics, Department of Mechanical Engineering and Aeronautics, University of Patras, \\ 26504 Patras, Greece \\ Correspondence should be addressed to P. Koutmos; koutmos@mech.upatras.gr
}

Received 29 April 2013; Revised 13 July 2013; Accepted 16 July 2013

Academic Editor: Constantine D. Rakopoulos

Copyright (C) 2013 G. Paterakis et al. This is an open access article distributed under the Creative Commons Attribution License, which permits unrestricted use, distribution, and reproduction in any medium, provided the original work is properly cited.

\begin{abstract}
The work presents comparisons of the flame stabilization characteristics of axisymmetric disk and 2D slender bluff-body burner configurations, operating with inlet mixture stratification, under ultralean conditions. A double cavity propane air premixer formed along three concentric disks, supplied with a radial equivalence ratio gradient the afterbody disk recirculation, where the first flame configuration is stabilized. Planar fuel injection along the center plane of the leading face of a slender square cylinder against the approach cross-flow results in a stratified flame configuration stabilized alongside the wake formation region in the second setup. Measurements of velocities, temperatures, $\mathrm{OH}^{*}$ and $\mathrm{CH}^{*}$ chemiluminescence, local extinction criteria, and large-eddy simulations are employed to examine a range of ultralean and close to extinction flame conditions. The variations of the reacting front disposition within these diverse reacting wake topologies, the effect of the successive suppression of heat release on the near flame region characteristics, and the reemergence of large-scale vortical activity on approach to lean blowoff (LBO) are investigated. The crosscorrelation of the performance of these two popular flame holders that are at the opposite ends of current applications might offer helpful insights into more effective control measures for expanding the operational margin of a wider range of stabilization configurations.
\end{abstract}

\section{Introduction}

The requirements in the design of fuel flexible, low emissions and versatile transportation and power generation systems are placing heavy demands on the development and performance of current combustion devices $[1,2]$. As a result the pursuit of new combustion concepts or modes is ongoing and a significant part of the overall design effort is devoted to maintain satisfactory flame stability and efficient emission targets $[2,3]$.

Over the years lean premixed combustion has gained popularity and has become widely exploited as it addresses some of the above issues over a range of applications [24]. However, the anticipated operation of future combustors under increased loads and mixing and burning rates may result in complications such as sensitivity to mixing, low reaction and heat release rates, extinctions, and instabilities [3-5].
Partially premixing and stratifying the reactive mixture is becoming an increasingly widespread strategy for burner design in the effort to expand the stability margin of the lean fully premixed concept and at the same time meet these combined requirements $[2,4-6]$. The effective control of spatially varying local fuel-air ratio distributions within the combustor offers significant operational flexibility. Leaner regions increase efficiency and reduce pollutant formation, and richer mixtures improve ignition performance and allow for better control of instabilities. However nonuniformities in the local fuel-air ratio can also lead to spatially varying combustion performance with implications that have to be addressed in relation to the local turbulent chemistry, the stabilization method, and its interaction with the stability margin (lean blow-out (LBO)) of the particular flame configuration [4-7].

Evidently due to the complexity of the above described phenomena, better knowledge of the details of the turbulent 
transport and reaction processes over a range of operating conditions from lean to ultra-lean and approaching blowoff (LBO) is important, if a full exploitation or even extension of the operational margin is to be realised (e.g., $[4,6-8]$ ). A range of systematic experiments have been performed to study the structure of such flame configurations (e.g., [4-8]) along with a number of supporting simulations that employ various levels of complexity for the turbulence-chemistry treatment and usually involve adaptive local gridding within a time-dependent procedure (e.g., [9]). Phenomenological analyses (e.g., $[2,6,8])$ and global correlations (e.g., $[3,10])$ are also utilized to complement the overall development effort and relieve the burden and cost involved in the multiscalar approaches.

These experimental and computational works have so far produced detailed descriptions of the ultra-lean behaviour in traditional stabilizers such as fully premixed axisymmetric (e.g., [3, 4, 7, 8]) and slender two-dimensional (e.g., [6, 11]) bluff-body arrangements. However, stratified bluff-body flames operated at ultra-lean and near blow off conditions have not been investigated as extensively. Moreover the growing combination of burner and mixture placement setups in lean combustors has increased current interest for a more complete description of such flame types. Therefore comparative examinations of the performance of the axisymmetric versus the slender two-dimensional bluff-body stabilization configuration under stratified conditions are warranted; these could provide useful information regarding the more effective control, exploitation, or even extension, for example, through appropriate placement of pilot injection, of the operational margin under both setups (e.g., $[2,4,6,11,12]$ ).

This work then compares the ultra-lean performance and topology characteristics of propane flames stabilized in an axisymmetric, double cavity fuel-air premixer/disk arrangement [13] and a counterinjected 2D slender square cylinder [11] under stratified conditions. The above geometries were chosen because these comprise two of the most popular stabilizers, at the opposite end of the field of practical applications, and comparisons of their limiting performance could provide useful directions in the design process for a wide range of configurations. Identification of the individual characteristics such as, for example, flame and recirculation lengths, flame front topologies, turbulence levels, vortical activity, and under successively weakening stabilization within the same geometry arrangement may help determine suitable methodologies to extend stability in a fashion tailored not only for the particular flame geometries but also for a range of intermediate variants, upgrades, or retrofits $[2,4$, 12]. Furthermore both of these setups have been systematically investigated under fully premixed conditions and their operation under stratified conditions could then be directly contrasted against such a range of well documented data.

Measurements of turbulent velocities, temperatures, chemiluminescence imaging of $\mathrm{OH}^{*}$ and $\mathrm{CH}^{*}$, flame front visualization, and gas analysis provided information for lean, ultra-lean, and close to blowoff flames. Supporting large-eddy simulations were also undertaken employing the thickened flame model $[14,15]$ and a nine-step mechanism for propane combustion $[11,13]$ to complement the experimental effort and assist in the more complete interpretation of the optical measurements.

The combined methodology helped to elucidate some of the parametric characteristics of these diverse flame topologies and improve understanding regarding the differences and similarities between these two stabilizers when operated with a stratified inlet mixture as opposed to a fully premixed operation. In addition, the local extinction behavior was examined by using the combination of three, measured or computed, parameters, namely, a local Karlovitz number, a local temperature threshold, and the ratio of the $\mathrm{OH}^{*}$ / $\mathrm{CH}^{*}$ chemiluminescence signature. The credibility of this parameterization is appraised, where possible, by using both measurements and simulations.

\section{Flame Stabilization Configurations}

The two burner setups are shown in Figures 1(a), 1(b), 1(c), 1(d), 1(e), and 1(f). In the first configuration stratified flames, established by staged fuel-air premixing in a double-cavity arrangement (Figure 1(c)) formed along three concentric disks and stabilized in the vortex region of the afterbody $\mathrm{A}$ $\left(D_{b}=0.025\right)$ under the additional action of a coflowing swirl stream (Figure 1(b)) are investigated. The combustion tunnel and the studied burner are similar to those reported in [13] and have been optimized through a series of systematic tests [16]. Fuel is supplied through the central shaft $\left(D_{p}=\right.$ $0.010)$ that is running along the central air supply tube $\left(D_{c}=\right.$ 0.052 ) into the internal hollow of the active bluff-body disk B (Figure 1(c)). Then it is injected through an annular $1 \mathrm{~mm}$ slot into the primary fuel air-mixing cavity and is mixed with the central air supply. This maintains an afterbody equivalence ratio gradient between a $\Phi_{\min } \approx 0.35$ to 0.20 and a $\Phi_{\max } \approx$ 0.98 to 0.5 , depending on flame condition, as measured with flame ionization detection from local gas samples at the afterbody exit (Figure 1(d)). Limiting conditions approaching blowoff were obtained by reducing the fuel level which then decreased the peak $\Phi$ at the primary zone inlet. In all cases a regulated stratified reactive mixture profile is attained across the most favourable position for leading edge flame front stabilization at the disk burner rim (Figure $1(\mathrm{~d})$ ). The simulated cavity flows for a lean flame at $S=1.0$ are shown in Figure 1(d) to illustrate the overall flow patterns obtained within and at the exit of the premixer system; these remain largely unaltered between lean and ultra-lean operation. The global $\Phi$, based on the total mass flows of fuel and central air together with the range of investigated conditions and parameters, is shown in Table 1 . The blockage ratio (BR) in the afterbody annular jet supplying the primary zone is $\mathrm{BR}=\left(D_{b} / D_{c}\right)^{2}=0.23$ [13].

The second setup involves the study of partially premixed and stratified flames established by planar injection (discrete jets of small aspect ratio) of propane along the center plane of the leading face of a slender square cylinder. Propane is supplied through the cylinder ends, issued through 135 holes of $1 \mathrm{~mm}$ diameter spaced at $0.5 \mathrm{~mm}$ apart on the symmetry plane over a width of $200 \mathrm{~mm}$ along the upstream face of the square, and reaction is stabilized within and adjacent to the downstream wake formation region (Figures 1(e) and 


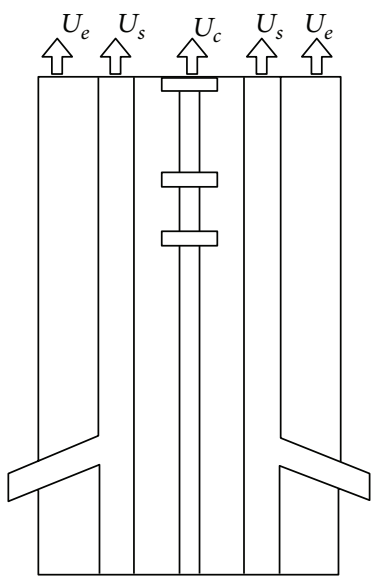

(a)

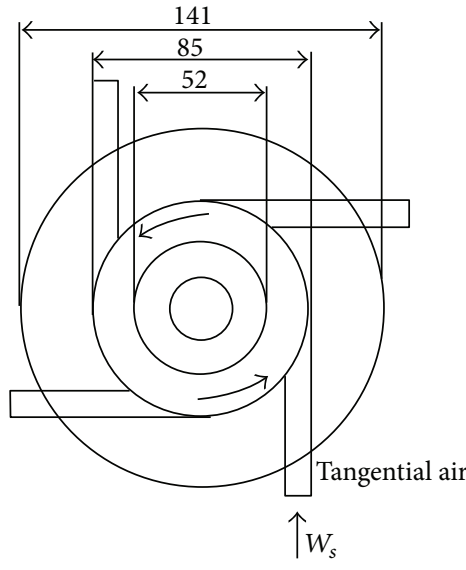

(b)

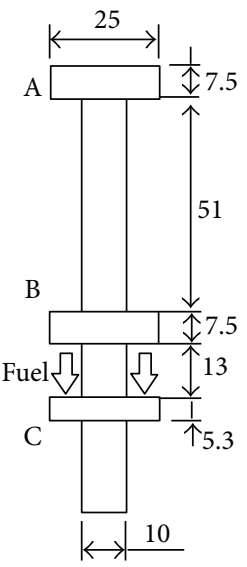

(c)

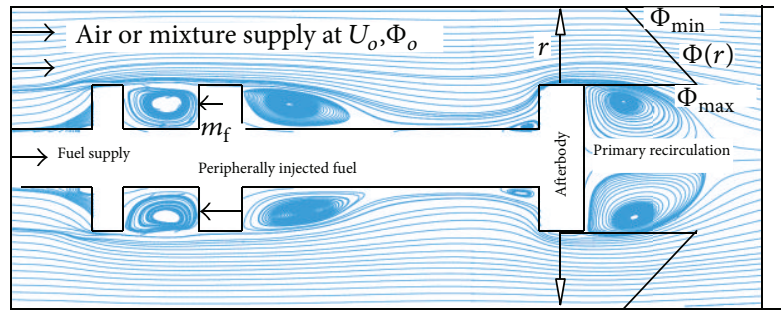

(d)

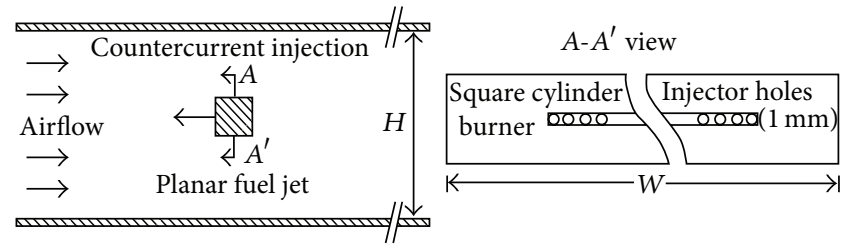

(e)

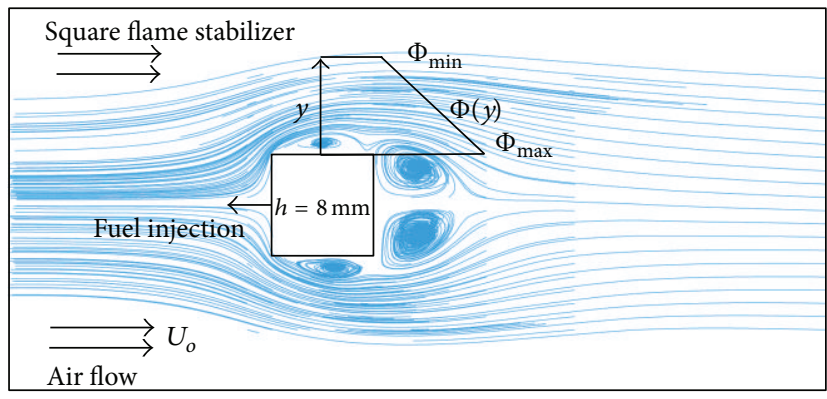

(f)

Figure 1: Axisymmetric disk ((a), (b), (c), and (d)) and slender square ((e), (f)) burner arrangements, including respective flow patterns, fuel-air placements, and mixture stratifications at inlet to the stabilization regions.

$1(\mathrm{f}))$. The level of stratification across the square flanks is controlled by the velocity ratio between the fuel injection and the approach cross-flow with peak levels ranging from 1.6 to 0.6 . The blockage ratio of the square burner in the twodimensional tunnel was $\mathrm{BR}=h / H=0.10$ [11]. The Reynolds numbers of the cold flow were maintained at 7980 and 5800 for the disk and the square, respectively.

A high swirl case was chosen for study in the disk configuration. The geometric swirl number, $S,(S=1.00$ for this investigation), used for the quantitative representation of swirl intensity, was expressed here as the ratio of integrated (bulk) tangential to primary axial air velocities $\left(W_{s} / U_{s}\right)$, measured through LDV in the coflow swirl stream. Square burner flames formed by counterinjection with a low fuelto-approach-air velocity ratio operated toward the lean LBO limit were chosen for the second case. Under both cases strong recirculation regions assist in flame stabilization under high stretch rates leading to a spread in the disposition of the flame front alongside the wake flanks. The wake development in the square is affected by periodically asymmetric vortex shedding with strength that drastically varies between isothermal and low or high fuel injection reacting conditions $[6,11,12,17]$. On the other hand the axisymmetric disk exhibits low frequency vortex rollup from the rim periphery, which is again stronger under isothermal conditions [13, 18]. The interaction of the locally evolving extinction process with the underlying turbulent wake dynamics presents several differences between the above two flameholders (e.g., [4, 6$8,12,13,17])$. This facilitates a useful evaluation of their different behaviors under ultra-lean operation and allows for an appraisal of the adopted modelling methodology.

All fuel flows were regulated by Bronkhorst MV-304/306 High-Tech mass flow controllers. Necessary supply valves, the ignite, and a flame-out detection device were used and were all controlled electronically by a central unit. 
TABLE 1: Operating conditions and parameters.

\begin{tabular}{lccccccc}
\hline Case & $\delta(\%)$ & $U_{\text {Fuel }}$ & $T_{\max }(\mathrm{K})$ & $L_{f} / D_{b}$ & $L_{R} / D_{b}$ & $\Phi_{\text {Global }}$ & $\mathrm{Ka}$ \\
\hline Disk & & & & & & & \\
$\quad$ Lean & 50 & 1.43 & 1860 & 5.6 & 1.43 & 0.147 & 6 \\
$\quad$ Ultralean & 18 & 1.12 & 1732 & 5.2 & 1.36 & 0.115 & 18.5 \\
$\quad$ Near LBO & 6 & 1 & 1508 & 4.4 & 1.28 & 0.102 & 47 \\
Square & & & & & & & \\
$\quad$ Lean & 94 & 1.24 & 2050 & 8.5 & 5.5 & 0.031 & 15 \\
$\quad$ Ultralean & 65 & 1.05 & 1850 & 3.5 & 3.5 & 0.025 & 35 \\
$\quad$ Near LBO & 14 & 0.73 & 1600 & 1.4 & 1.45 & 0.018 & 72 \\
\hline
\end{tabular}

(1) $\delta$ is percent deviation from LBO, $\delta=\left(m_{\text {Fuel }}-m_{\text {Fuel,LBO }}\right) / m_{\text {fuel,LBO }}(\%)$, ( $m_{\text {fuel,LBO }}$, fuel flow at lean blowoff $) . U_{\mathrm{FBO}}=0.95 \mathrm{~m} / \mathrm{s}$ for disk and $0.64 \mathrm{~m} / \mathrm{s}$ for square.

(2) $T_{\max }:$ maximum measured wake temperature.

(3) $L_{f}$ : visible flame length; $L_{R}$ : measured recirculation length.

(4) Central air supply velocity for disk, $U_{c}=4.87 \mathrm{~m} / \mathrm{s}$ and $\mathrm{Re}_{\mathrm{Db}}=7980$ for all cases, coflow velocities in annular swirl stream at $S=1.0, U_{s}=W_{s}=$ $10.5 \mathrm{~m} / \mathrm{s}$, surrounding shielding stream velocity, $U_{e}=10 \mathrm{~m} / \mathrm{s}$. Approach airflow for square, $U_{\infty}=6.7 \mathrm{~m} / \mathrm{s}$ and $\mathrm{Re}_{H}=5800$ for all cases.

(5) $\Phi_{\text {Global }}$, global equivalence ratio, based on mass flows of injected fuel and, (a) air supply in the central pipe for the disk, or (b) approach airflow over the tunnel height for the square.

(6) $T_{\max }$ location for the disk was at $\left(x / D_{b}, r / D_{b}\right)=(0.4,0.45)$, while for the square this was at $(x / h, y / h)=(0.7$ to $1.0,0.9)$.

Flame nomenclature in both cases is denoted on the basis of the proximity to $\mathrm{LBO}$, defined by $\delta=\left(m_{\text {Fuel }}-\right.$ $\left.m_{\text {Fuel,LBO }}\right) / m_{\text {Fuel,LBO }}(\%)$, where $m_{\text {Fuel,LBO }}$ is the fuel flow at lean blow-out. The global $\Phi$ based on the total mass flows of fuel and approach air together with the range of investigated conditions and parameters is included in Table 1.

\section{Experimental Methods}

The burners have been previously investigated under isothermal operation to establish the mixing topologies that sustain the reacting fields presented below [11, 13, 16, 19]. Mean and turbulent velocities, temperatures, and $\mathrm{OH}^{*}$ and $\mathrm{CH}^{*}$ chemiluminescence (CL) images were obtained using a two-component LDV system, thin digitally compensated thermocouples, and a chemiluminescence imaging system (LaVision Flame Master consisting of a CCD camera, an image intensifier IRO unit, camera optical filter at $307 \pm 10 \mathrm{~nm}$ and achromat lens for $\mathrm{OH}$ imaging).

Transverse profiles of the time-averaged mean and turbulent streamwise and cross-stream velocities and statistics were obtained with a two-component 2 Watt Argon-Ion laser, fiber optics linked to TSI transmitting and receiving optics and described in detail in [19]. The fuel and air flows were seeded with dried magnesium oxide powder (approximately $5 \mu \mathrm{m}$ nominal diameter before agglomeration) dispersed by two purpose-built cyclone separators. In order to minimize bias errors due to unequal particle densities in the fuel and air streams, the two flows were seeded separately, with rates of particles in proportion to the flow rates in each one. Extreme deviations were determined by seeding only one of the two streams. The filtered Doppler signals were processed by a TSI frequency counter (1980B). Mean and statistical values were postprocessed from 20480 data weighed by the time between particles to correct for velocity bias. Velocity spectra were obtained by sampling at $4 \mathrm{kHz}$ and performing a fast fourier transform (FFT) on 20 sets of 1024 values [11, 19].

Temperatures were measured with $\mathrm{Pt}-\mathrm{Pt} / 10 \% \mathrm{Rh}$ uncoated beaded $S$-type 25 to $75 \mu \mathrm{m}$ thermocouples. A twin bore ceramic cladding of $130 \times 0.9 \mathrm{~mm}$ with $0.2 \mathrm{~mm}$ capillary holes, oriented along the flow, supported the stem. The T/C output was interfaced to a DaqTemp 7A Omega card. The thermocouple acts as a low-pass filter with a response of the order of 50 Hertz for, for example, $100 \mu \mathrm{m}$ wires that depend on local parameters (position, temperature, junction geometry and material, gas velocity, conductivity, heat capacity and density, and flame structure). Here, using an FFT algorithm, the signal's frequency spectrum was estimated, the amplitude and the phase were corrected, and the signal was transformed back to the time domain according to the methodology described in [20], so that about $60 \%$ of the temperature fluctuations spectrum could be calculated $[11,19,20]$. No correction was applied for radiation, but for propane flames and uncoated wires, the systematic error in the mean can be up to $10 \%$ at $1900 \mathrm{~K}$ [19]. Mean and rms temperatures were derived at a sampling frequency of $400 \mathrm{~Hz}$. Maximum uncertainties in the velocities were less than $8.5 \%$ in the mean and less than $15 \%$ in the rms. Uncertainties in the estimation of the thermocouple time constant are rather unimportant for the mean temperature, but according to the present compensation procedure may affect the variance by between 25 and $35 \%$.

Chemiluminescence measurements of the electronically excited $\mathrm{OH}^{*}$ and $\mathrm{CH}^{*}$ radicals are frequently employed to identify the topology of the flame front, since, for example, the electronically excited $\mathrm{OH}^{*}$ exists mainly in the flame front region (e.g., $[4-7,12])$. Image acquisition and data reduction were performed using the Davis 8.0 software from LaVision. The background images taken under the same integration time and gain were subtracted from the originals. Averages were produced from 300 instantaneous images recorded at 16 to 60 frames per second depending on the binning and the interrogation window. The signal-to-noise ratio of the instantaneous images was better than $8: 1$, and the exposure time was $2.1 \mathrm{~ms}$ under a single frame mode operation. The maximum CCD chip resolution was $1626 \times 1236$ pixels. Intensifier gate times of the order of $100 \mu$ s were used for the measurements with a gain up to about $85 \%$. Since the CCD is combined with an intensifier, the effective exposure is determined by the image intensifier gate, which typically is of the order of several nsecs, whilst the image collection also depends on the attainable CCD recording rate. The camera exposure is a temporal envelope that integrates the phosphor emission of the intensifier whilst maintaining an exposure time greater than the sum of the delay and the gate times.

A direct qualitative comparison between the time-mean simulated $\mathrm{OH}$ mole fraction fields with the experimentally measured mean $\mathrm{OH}^{*}$ chemiluminescence fields is not immediately possible. This is because the experimental measurements are effectively a $2 \mathrm{D}$ image of projected line of sight measurements from a cylindrically symmetric process (in the mean), whilst the numerical results are a $2 \mathrm{D}$ axisymmetric 
slice through the axis of symmetry of the cylindrically symmetric process. To compare the two different datasets, a transformation process is required to be applied to one or both of the datasets. As the time-average flame is axisymmetric, the location of the reaction zone can therefore be observed from converting the ensemble average chemiluminescence image (obtained from a set of 300 images) with an Abel transform. Three-point Abel deconvolution formulations given by Dasch [21] were used to extract two-dimensional information from the ensemble average chemiluminescence images, as the three-point Abel inversion algorithm was previously found to be more accurate and efficient when compared to onion peeling, filtered backprojection, and two-point Abel deconvolution methods, as demonstrated in [7, 21].

A quartz microprobe was used to obtain samples at the afterbody annular exit, and flame ionization detection (employing a Signal 3010 MINIFID device) was employed to measure the concentration of the hydrocarbon at this position and allow the evaluation of the equivalence ratio levels with an uncertainty of the order of $4 \%$. Global exhaust emissions could also be measured by extracting flue gases and measuring bulk species $\left(\mathrm{NO}_{x}, \mathrm{CO}, \mathrm{CO}_{2}, \mathrm{O}_{2}\right.$, and $\left.\mathrm{C}_{x} \mathrm{H}_{y}\right)$ concentrations with a Kane-May KM9106 Quintox flue gas analyzer further downstream of the burner [11, 13].

\section{Simulation Model Formulation}

4.1. Aerodynamic Model. The flames were simulated with the ANSYS Fluent 14.0 (ANSYS Inc.) software [22]. It offered mesh adaption flexibility near the fuel injectors and facilitated the exploitation of reduced chemistry within the context of an adequate modeling scheme for the turbulent reactions. Within the Large Eddy Simulation procedure employed here, the flow variables, $F$, can be decomposed into resolvable $\widetilde{F}$ and subgrid, $F^{\prime}$, scale quantities using a Favre-weighed filter, $\widetilde{F}=\overline{\rho F} / \bar{\rho}$. The equations describing the resolvable flow quantities are, for example, $[9,13,14,22]$.

Continuity, Momentum is

$$
\begin{gathered}
\frac{\overline{\partial \rho}}{\partial t}+\frac{\partial\left(\bar{\rho} \widetilde{u}_{i}\right)}{\partial x_{i}}=0 \\
\frac{\partial \bar{\rho} \widetilde{u}_{i}}{\partial t}+\frac{\partial\left(\bar{\rho} \widetilde{u}_{i} \widetilde{u}_{j}\right)}{\partial x_{j}}= \\
=\frac{\partial \bar{p}}{\partial x_{i}}+\frac{\partial}{\partial x_{j}}\left[\mu\left(\frac{\partial \widetilde{u}_{i}}{\partial x_{j}}+\frac{\partial \widetilde{u}_{j}}{\partial x_{i}}\right)\right] \\
-\frac{\partial \tau_{i j}^{a}}{\partial x_{j}}+\left(\Delta \bar{\rho}_{\infty}\right) g_{i}
\end{gathered}
$$

and Scalars are

$$
\begin{aligned}
\frac{\partial \bar{\rho} \tilde{Y}_{k}}{\partial t}+\frac{\partial\left(\bar{\rho} \tilde{u}_{j} \tilde{Y}_{k}\right)}{\partial x_{j}}= & \frac{\partial}{\partial x_{l}}\left(\bar{\rho} D_{k} \frac{\partial \tilde{Y}_{k}}{\partial x_{l}}\right) \\
& -\frac{\partial J_{l}}{\partial x_{l}}+\bar{\rho} \tilde{\dot{\omega}}_{Y_{k}}, \quad k=1, N .
\end{aligned}
$$

The solution of an energy equation completes the above system $\left(\rho, \mu, T, u_{i}\right.$, and $Y_{k}$ are the density, viscosity, temperature, velocity and species composition of the gas, and $i=$
$1,2,3$ in a Cartesian system $(x, y, z)) \cdot \tau_{i j}^{\alpha}=\tau_{i j}-1 / 3 \tau_{i j} \delta_{i j}$ is the anisotropic part of the subgrid stress tensor, $\tau_{i j}$, with the isotropic part of the viscous and subgrid stress being adsorbed into the pressure. The subgrid stresses are modeled as $\tau_{i j}^{\alpha}=-2 \mu_{\text {sgs }} \widetilde{S}_{i j}$, where $\mu_{\text {sgs }}=\tilde{\rho}\left(C_{s} \bar{\Delta}\right)^{2}\left(2 \widetilde{S}_{i j} \widetilde{S}_{i j}\right)^{1 / 2}, \widetilde{S}_{i j}$ is the resolvable strain tensor and $\Delta$ is the filter width related to the local mesh spacing, $\Delta=\sqrt[3]{\Delta x_{i} \Delta y_{i} \Delta z_{i}}$. A gradient assumption is used for the subgrid scalar flux, $J_{k}=\bar{\rho}\left(\widetilde{u_{l} Y_{k}}-\widetilde{u_{l}} \widetilde{Y_{k}}\right)=$ $-\left(\mu_{\text {sgs }} / \mathrm{Sc}_{\mathrm{sgs}}\right)\left(\partial \widetilde{Y_{k}} / \partial x_{l}\right)$, where $\mathrm{Sc}_{\mathrm{sgs}}$ is the subgrid Schmidt number. Turbulent transport is modelled with the dynamic Smagorinsky model (e.g., $[9,11,22])$ with bounded $C_{s}(0<$ $\left.C_{s}<0.23\right)$. Pressure-velocity coupling was handled via a time-dependent SIMPLE algorithm (e.g., [9, 22]). A secondorder accurate time discretization was used, and the time step was chosen to maintain a maximum Courant number between 0.4 and 0.6 . Rms values were computed from the resolved scale quantities, including the contributions from the subgrid scale stresses. The simple P-1 radiation model available in the software (ANSYS Inc.) was adopted in the simulations (although no correction for radiation was applied to the temperature measurements; its use in the simulations allows some flexibility in future processing of the present data).

The employed commercial software has frequently been used to investigate with success premixed flame stabilization in complex bluff-body configurations under stable (e.g., [23]) and approaching blowoff [24] conditions in a fashion similar to the present study. One should nevertheless exercise great caution in interpreting the results obtained regarding the behaviour and development of the flame front at the final stages toward LBO; centers of excellence in combustion studies worldwide are currently trying to develop such a capability and capture this complex phenomenon with sufficient accuracy [23]. Notwithstanding the above comments, here every effort was made to improve the basic capability of the software through use (a) of the higher order differencing schemes available within the software for LES, (b) of a well-established turbulence-chemistry closure such as the thickened flame model, (c) of the extended nine-step chemical scheme for propane chemistry, and (d) through careful mesh refinement in the main reaction zone. With these improvements the performance of the software was deemed satisfactory in providing useful information at ultralean and near LBO conditions, and this was tested where possible against the present measurements.

4.2. Combustion Model. To represent the mixed regime combustion that may result from the various fuel settings the thickened flame model (TFM) (e.g., [14, 15, 22, 23]) was employed. This formulation was coupled to a nine-step reduced scheme for propane (i.e., $\mathrm{Rxn} \mathrm{1:} \mathrm{C}_{3} \mathrm{H}_{8}+\mathrm{O}_{2}+\mathrm{OH}+$ $\mathrm{H} \rightarrow 3 \mathrm{CO}+5 \mathrm{H}_{2}$, Rxn 2: $\mathrm{CO}+\mathrm{OH} \leftrightarrow \mathrm{CO}_{2}+\mathrm{H}$, Rxn 3: $3 \mathrm{H}_{2}$ $+\mathrm{O}_{2} \leftrightarrow 2 \mathrm{H}_{2} \mathrm{O}+2 \mathrm{H}$, Rxn 4: $2 \mathrm{H}+\mathrm{M} \rightarrow \mathrm{H}_{2}$, Rxn 5: $\mathrm{H}_{2}+\mathrm{O}_{2}$ $\leftrightarrow 2 \mathrm{OH}, \mathrm{Rxn} 6: \mathrm{C}_{3} \mathrm{H}_{8}+\mathrm{O}+\mathrm{OH}+\mathrm{H} \rightarrow \mathrm{C}_{2} \mathrm{H}_{2}+\mathrm{CO}+\mathrm{H}_{2} \mathrm{O}$ $+3 \mathrm{H}_{2}, \mathrm{Rxn} 7: \mathrm{C}_{2} \mathrm{H}_{2}+\mathrm{O}_{2} \leftrightarrow 2 \mathrm{CO}+\mathrm{H}_{2}$, $\mathrm{Rxn} 8: 2 \mathrm{O}+\mathrm{M} \leftrightarrow \mathrm{O}_{2}$, $\mathrm{Rxn}$ 9: $\left.\mathrm{O}_{2}+\mathrm{H} \leftrightarrow \mathrm{O}+\mathrm{OH}\right)$. This represents an extension of the mechanism described in $[11,13,16]$, now including the radical $\mathrm{OH}$ to facilitate more direct comparisons with the measured 
chemiluminescent $\mathrm{OH}^{*}$ and has been tuned to reproduce the flame speed over a section of the lean $\Phi$ range as described in [11]. All the species are explicitly solved on the computational grid, and it is likely that intermediate radicals with shorter time scales might not be properly resolved within the context of the employed refinement. In the present low Reynolds number laboratory flames, the exploitation of the above combination of turbulence models and chemistry scheme can be considered attractive, in providing a more detailed and extensive description of the developing flame front properties while also allowing for a more direct comparison with optical experimental results.

The averaged reaction rate source terms were computed with the ISAT algorithm (e.g., [7, 19, 22]). Previous measurements [11,13] have suggested that the lean flames lie in the thin reaction regime. The present ultra-lean flames are closer to the broken reaction zone boundary with Karlovitz numbers [1] $\left(\mathrm{Ka}=\tau_{\mathrm{ch}} / \tau_{\mathrm{k}}\right.$, where $\tau_{\mathrm{ch}}$ and $\tau_{\mathrm{k}}$ are the chemical and Kolmogorov timescales) of about 80 and local grid-based Karlovitz numbers (e.g., [9]) of higher values.

4.3. Local Extinction Monitoring Parameters. An effort was also made to examine the local extinction behaviour at near LBO conditions by considering the combination of three, measured or computed, parameters. First, the parameter $\Lambda=\mathrm{Ka} / \mathrm{Ka}_{\mathrm{ex}}$, computed from the transient simulations, was chosen, where $\mathrm{Ka}$ is the local Karlovitz number and $\mathrm{Ka}_{\mathrm{ex}}$ is the Karlovitz stretch factor for symmetric counterflow laminar flame extinction being a function of the local equivalence ratio with $\Lambda>1$ signifying local extinction (e.g. [2,10]).

Second, the computed statistical distribution of the temperature difference $\Delta T_{c}$ between the local instantaneous temperature, $T_{\text {inst }}$, and a chosen threshold temperature, $T_{\text {limit }}$, $\Delta T_{c}=T_{\text {inst }}-T_{\text {limit }}$ was evaluated, and local extinction is considered when $\Delta T_{c}<0$; the usefulness of a threshold temperature value has been demonstrated from the extensive experimental investigations on $\mathrm{C}_{3} \mathrm{H}_{8}$ flame extinction of reference [3]. For conditions close to the present experiment, values of $T_{\text {limit }}$ of around $1400 \mathrm{~K}$ have been reported for swirl, grid, and porous plate burners [3], while a similar range of limiting extinction temperatures was measured and computed in laminar counterflow setups [2]. Present tests in the simulations suggested a value between 1325 and $1350 \mathrm{~K}$.

Third, the trends in the variations of the ratio of the measured time-mean $\mathrm{OH}^{*} / \mathrm{CH}^{*}$ chemiluminescence signature, evaluated at selected locations close to the reacting flame front stabilization region, within the recirculation zone, at $x / D_{b}=0.44$ for the disk and at $x / h=1.6$ for the square, were examined for possible correlation with the proximity to blowoff. Although local extinction events are undoubtedly instantaneous phenomena, the proximity to LBO has routinely been assessed, for preliminary as well as practical studies, through time-averaged criteria, for example, Damkholer numbers, and so forth. Therefore the exploitation of the average $\mathrm{OH}^{*}$ to $\mathrm{CH}^{*}$ ratio cannot be excluded as a useful criterion. Furthermore, due to the complexity of the phenomena involved within time scales of a few milliseconds, apart from fast recording instrumentation, customarily more than one parameter has been examined to determine the flame condition, something that in most cases is and should be technical practice. Here the possibility of using the above parameters, in combination, as likely local markers for delineating reactive from nonreactive regions, in the present stratified flames with inlet mixture nonuniformities that place them neither in the nonpremixed nor in the fully premixed regime and the likelihood of extrapolating this behaviour to determine the proximity to LBO are tentatively explored.

4.4. Computational Details. Particular emphasis was given to the mesh distributions near the low aspect ratio injectors with respective dimensions, $(1: 25: 52 \mathrm{~mm}) \rightarrow$ (annular injection slot: afterbody: central pipe) for the disk and (1:8:80 mm) $\rightarrow$ (injection hole: square cylinder height: tunnel height) for the square (see Figure 1). The mesh for the disk extended over a $360^{\circ}$ sector, while for the square the resolved span was chosen to include seven injection holes plus one half spacing on each side, over a width of $1.75 \mathrm{~h}$, with symmetry boundary conditions at the lateral boundaries. The complete premixer cavities section was included in the mesh for the disk and the computations started well upstream of the complex premixer/burner system to incorporate the effects of the mean and turbulent inflow conditions measured at this position. Similarly the computations for the square started $7 D_{b}$ upstream of the burner face, where measured inlet velocity and turbulence profiles were available. Hybrid meshes were utilised, unstructured near the compound regions of the disk or the square injectors and the burner boundaries, and structured in other locations with expansion to the outlet, which was placed $15 D_{b}$ downstream of the bluff bodies. Typical meshes of 1.45 and 1.75 Mcells were employed for the disk and the square, respectively, in the basic runs. No-slip boundary conditions were used near the walls with the node close to the burner surface placed at $\Delta y / D_{b}=0.00175$, while the law of the wall was applied elsewhere. Inlet conditions were taken from measurements, while a static pressure boundary condition was applied at the outlet and all outflowing quantities were extrapolated from the interior node. The LES quality was checked by calculating the resolved fraction of the turbulence energy, $R_{k}=k_{\text {res }} /\left(k_{\text {res }}+k_{\text {sgs }}\right)$, with values up to $95 \%$ within the main reaction zones. Run times on $483.0 \mathrm{GHz}$ processors $(4$ XEON 5660) and $243.8 \mathrm{GHz}$ processors (4 i7) run in parallel were about 1.7 CPU hrs for the simulation of one through flow time $\left(\tau=D_{b} / U_{c}=5 \times 10^{-3} \mathrm{~s}\right)$ for the disk, while more computational time was necessary for the square due to the denser meshes utilized to resolve the discrete, small aspect ratio, series of injector holes. Time series of various quantities were collected for, for example, the disk over about $225 \tau$ and relevant statistics were postprocessed from this sample.

\section{Results and Discussion}

A description of the isothermal flow and the stable flame characteristics under operation away from the blow off limit region has been presented in references [11, 13, 16, 19] for the two burner configurations, respectively. The present work 


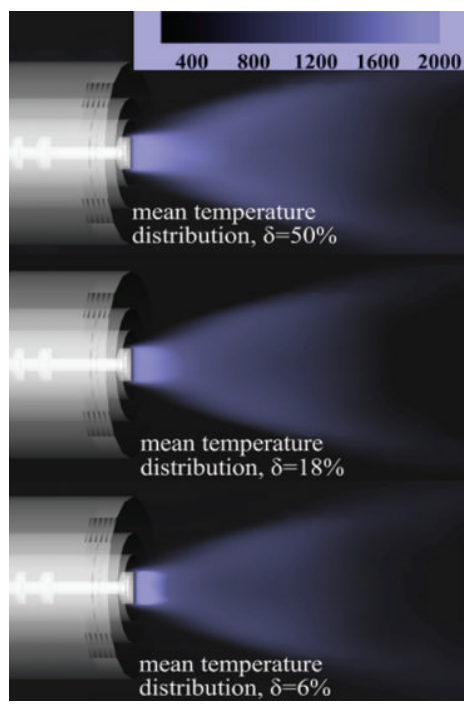

(a)

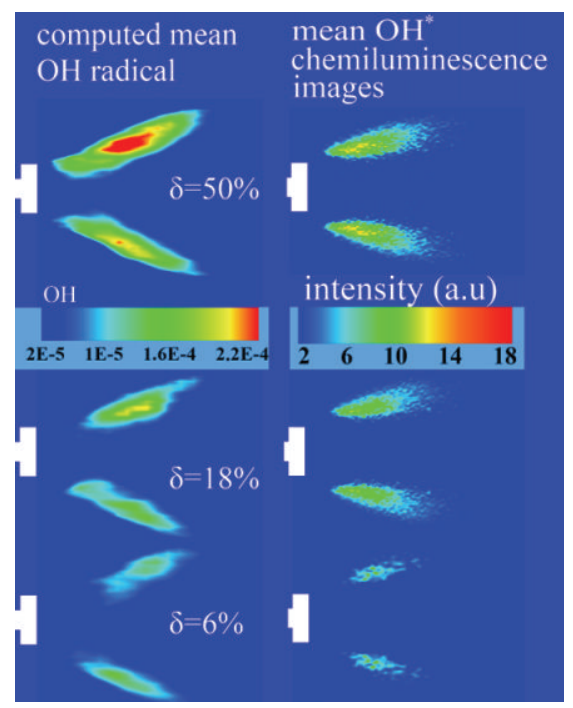

(b)

Figure 2: Simulated time-mean disk burner wake characteristics, (a) temperature, (b) $\mathrm{OH}$ mole fraction radical and $\mathrm{OH}^{*}$ chemiluminescence distributions for various ultra-lean flame settings.

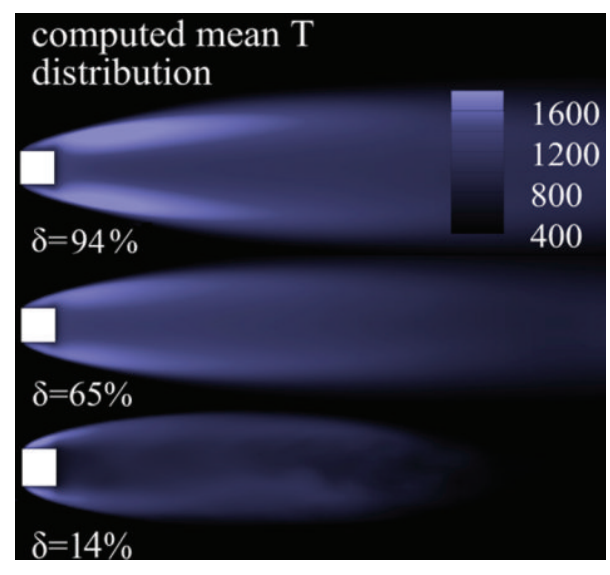

(a)

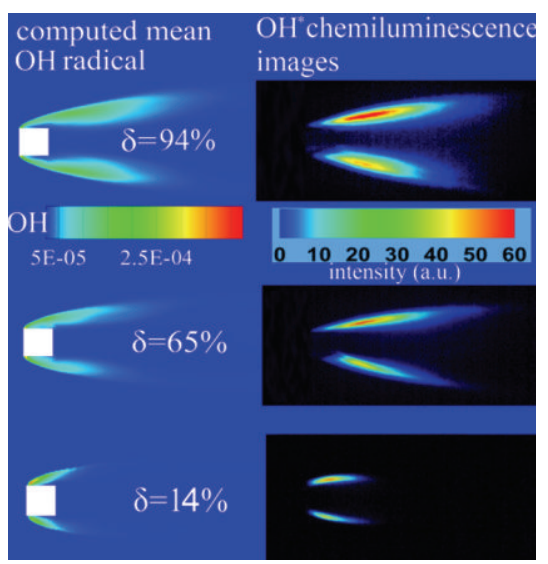

(b)

Figure 3: Simulated time-mean square burner wake characteristics, (a) temperature, (b) $\mathrm{OH}$ mole fraction radical and $\mathrm{OH}^{*}$ chemiluminescence distributions for various ultra-lean flame settings.

focuses on the comparative examination of the topologies obtained in the disk flame operated under a high swirl level $(S=1)$ and the square burner counterinjected with a low fuel-to-approach-air velocity ratio at ultra-lean conditions located near the LBO margin. The data presented for the two configurations are obtained for flame settings with successively reduced fuel flows, that is, at reduced $\delta$ levels (as indicated in Table 1).

An overall picture of the two reacting wake flows emerges in the simulated time-averaged patterns displayed in Figures 2 and 3, respectively, in the form of mean temperature and $\mathrm{OH}$ radical mole fraction contours together with the corresponding distributions of measured $\mathrm{OH}^{*}$ chemiluminescence. A peak temperature region is initially situated within and adjacent to the afterbody disk recirculation and extends up to $2.25 D_{b}$ with a conical growth of the hot wake at the fuel lean condition $(\delta=50 \%$, Figure 2(a)). At the leaner setting, the flame retains its conical shape, but the peak temperature envelope suffers a noticeable reduction with a moderate narrowing of the downstream wake spread. Closer to blowoff (at $\delta=6 \%$ ) the peak temperature region now recedes considerably closing in on the axis with the peak temperatures withdrawn toward the rim shear layers. The computed time-averaged $\mathrm{OH}$ mole fraction contour plot in Figure 2(b) also suggests that the leading edge of the toroidal flame front gradually weakens and detaches from the disk rim, while its trailing edge moderately retracts. The accompanying time-averaged $\mathrm{OH}^{*}$ chemiluminescence images in Figure 2(b) (Abel transformed images) corroborate to some extent the flame detachment but suggest a thinner reacting front with a much weaker activity particularly close to the disk axis. These plots imply that the present 


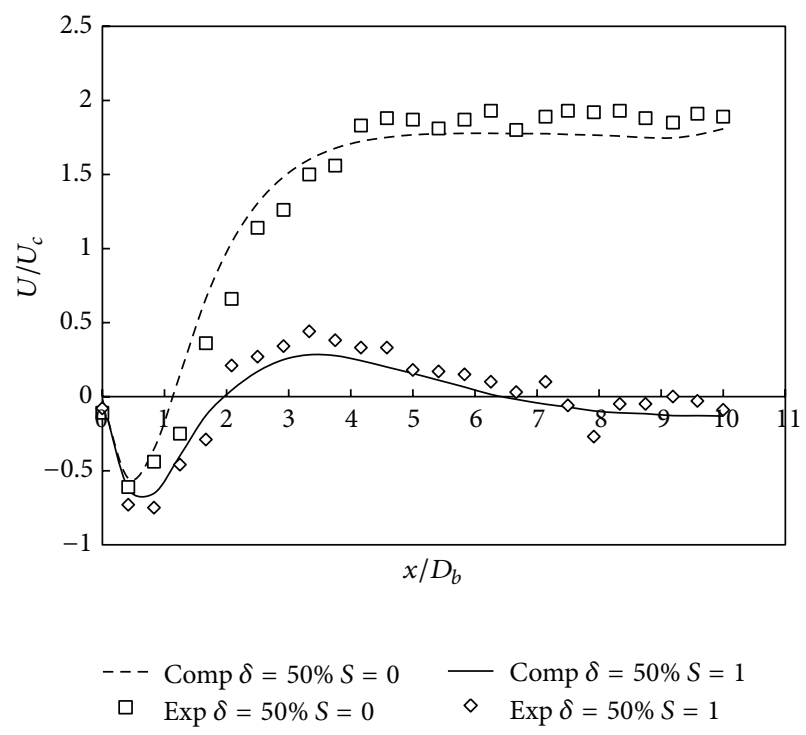

(a)

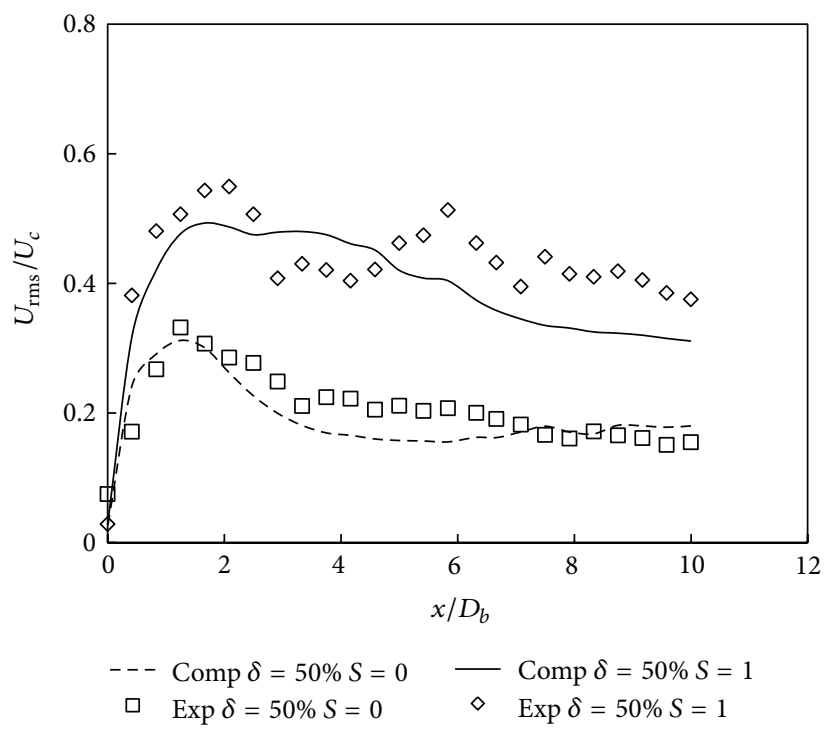

(b)

FIgURE 4: Comparisons between simulated and measured time-mean streamwise velocity (a) and turbulence intensity (b) distributions along the near wake of the disk burner.

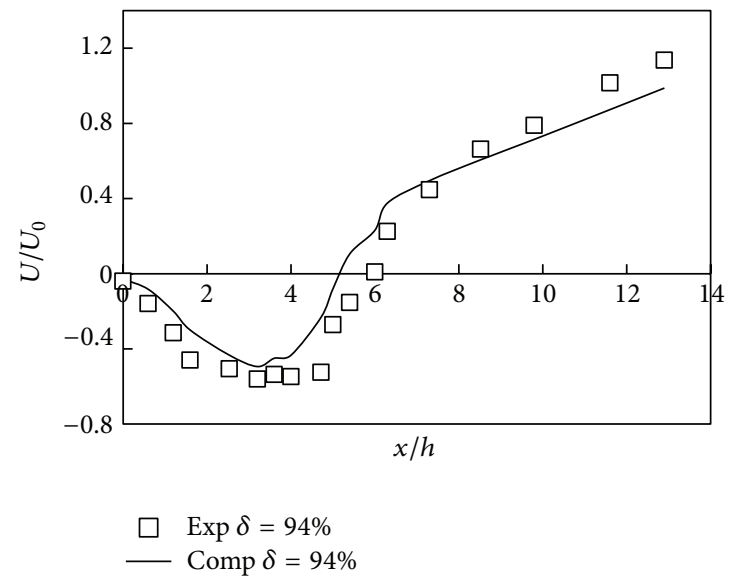

(a)

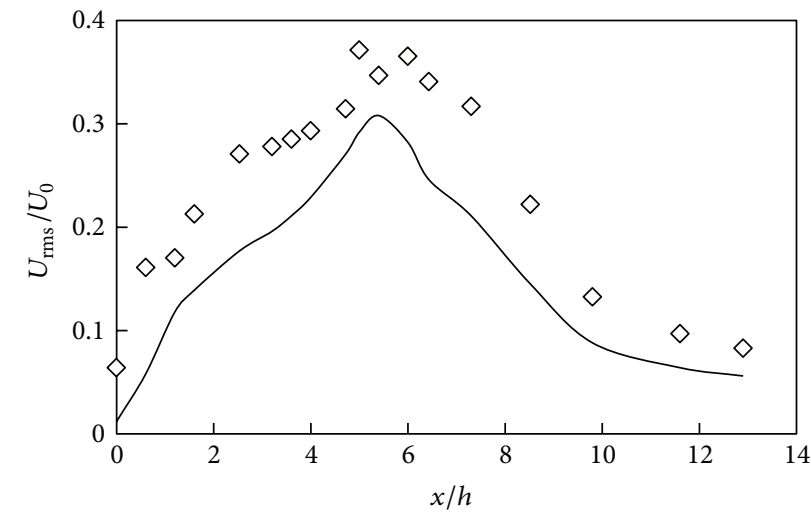

$\diamond \operatorname{Exp} \delta=94 \%$

— Comp $\delta=94 \%$

FIGURE 5: Comparisons between simulated and measured time-mean streamwise velocity (a) and turbulence intensity (b) distributions for the near wake of the square burner.

partially premixed disk flames behave somewhat differently than the fully premixed axisymmetric configurations burning methane that were visualized in [7] to drastically close in on the axis at the limiting lean operation; the disk flame disposition with the present leaner outward gradient inlet stratification follows closely the behaviour reported in [5] for similar stratification conditions.

Time-mean temperature contours for the square cylinder reacting wakes are presented in Figure 3(a) for medium, low, and limiting fuel injection velocities. The counterinjected configuration allows for partial premixing prior to ignition and stabilization around the cylinder flanks thus resulting in temperature shear layers that enclose the recirculation zone. Gradual fuel reduction here seems to lead to an attendant shortening of these flanking flame fronts, as illustrated by the computed time-mean $\mathrm{OH}$ radical contours and the included $\mathrm{OH}^{*}$ images in Figure 3(b). It should be noted that fuel injection directly into the vortex region would result in an altogether different reacting configuration of the nonpremixed type $[11,19]$.

The development of the near wake recirculation regions in terms of the measured and computed time-mean streamwise velocity and turbulence intensity distributions is shown in Figures 4 and 5 for the disk and the square burners, respectively, operating at lean conditions (Table 1). Good agreement is maintained in the axial velocity profile comparisons (Figure $4(\mathrm{a})$ ) at a range of conditions spanning the plane wake $(S=0)$ with lower turbulence levels and the 


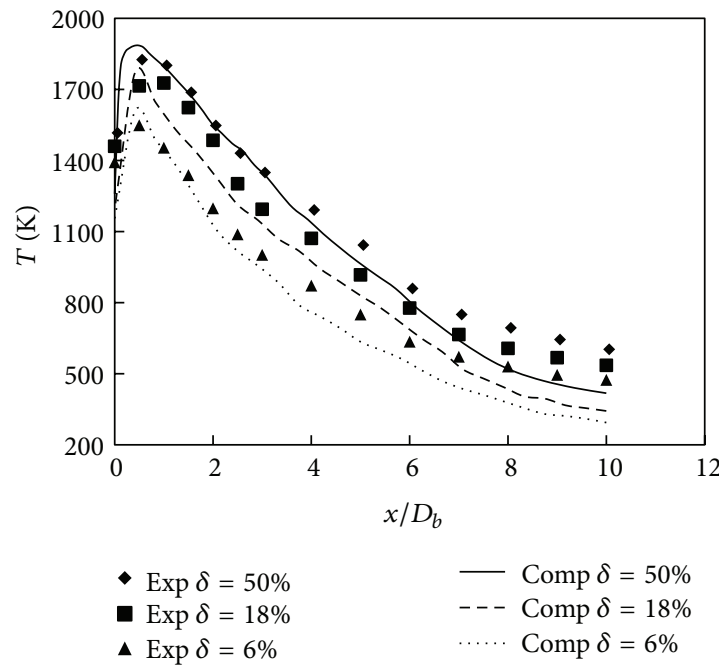

(a)

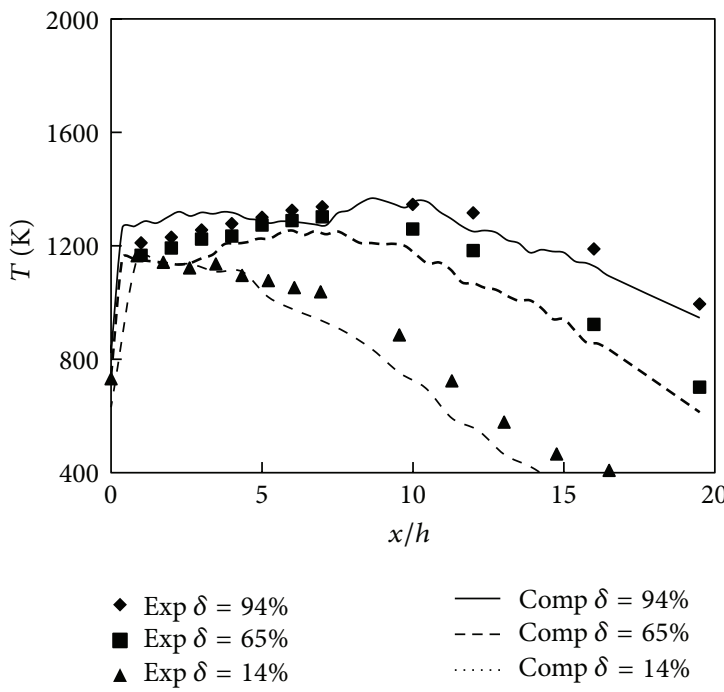

(c)

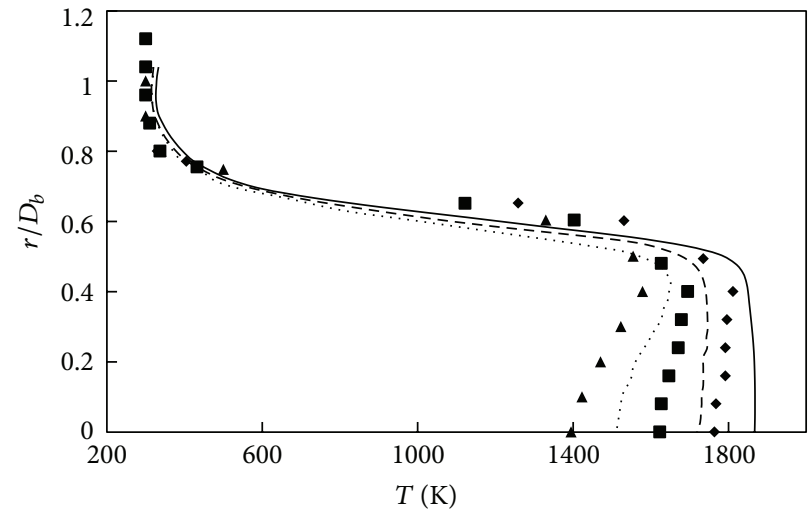

- $\operatorname{Exp} \delta=50 \%$

- $\operatorname{Exp} \delta=18 \%$

\ $\operatorname{Exp} \delta=6 \%$

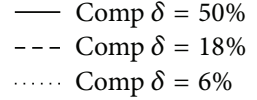

(b)

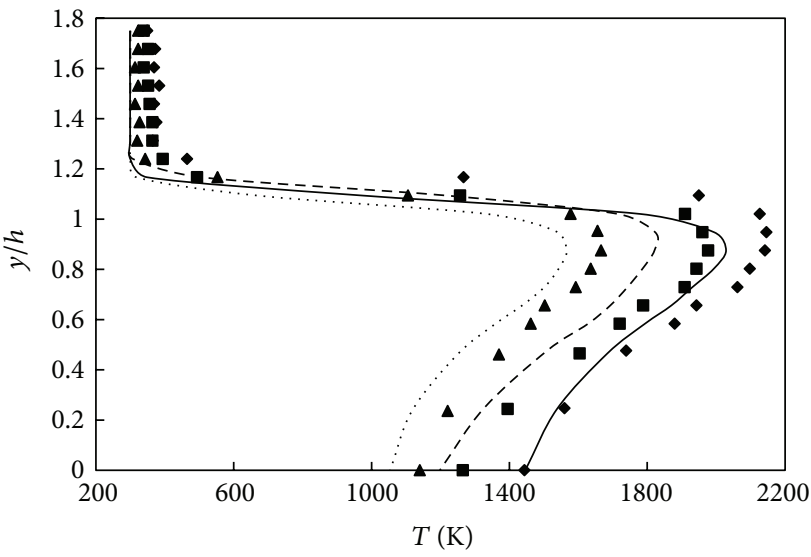

- $\operatorname{Exp} \delta=94 \%$

- $\operatorname{Exp} \delta=65 \%$

$\Delta \operatorname{Exp} \delta=14 \%$

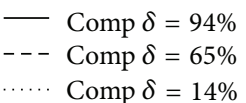

(d)

FIGURE 6: Comparisons between simulated and measured time-mean streamwise and cross-stream temperature distributions for disk ((a), (b)) and square ((c), (d)) burners, respectively. Cross-stream temperature distributions were taken at $x / D_{b}=0.4$ for the disk and at $x / h=0.6$ for the square.

higher swirl case $(S=1)$ with elevated turbulence levels (Figure $4(\mathrm{~b})$ ). In the disk wake, the recirculation lengths were found to vary from $1.43 D_{b}$ for the lean $(\delta=50 \%)$ to $1.28 D_{b}$ for the weaker $(\delta=6 \%)$ flame, respectively, gradually approaching the cold vortex zone length, and this trend was followed well by the simulations. On the other hand in the square burner the recirculation region was significantly elongated, in both measurements and simulations, by up to 5.5 times with respect to the cold wake, due to heat release and this effect is depicted by the extensive backflow region in the time-mean streamwise velocity profile comparisons shown in Figure 5(a). Maximum axial turbulence intensities are obtained for the disk at the higher swirl level of $S=1$ (Figure 4(b)) and are of similar magnitude to the intensities attained in the square burner flame (Figure 5(b)) justifying the choice of the higher swirl disk case for direct comparisons with the square burner configuration.
Time-mean temperature distributions for the disk and the square are shown in Figures 6(a), 6(b), 6(c), and 6(d) in the form of center-line (-plane) profiles along the wakes and radial (cross-stream) traverses taken close to the stabilizers, across the recirculation zone, respectively. The measured axial temperature variations (Figure 6(a)) are reproduced satisfactorily by the combined methodology in the near axisymmetric wake up to $x / D_{b}=4$, while discrepancies can be identified very close to the disk wall and in the downstream region where a faster wake development is computed. The lack of a more complete heat transfer modelling near the wall (apart from the use of the simple radiation model), the deficiencies of the TFM model in the vicinity of the wall due to a combination of inadequate mesh resolution, and likely shortcomings in the performance of the chemistry scheme there or the increased thermocouple measurement errors closer to the wall may be in part responsible for this discrepancy. 


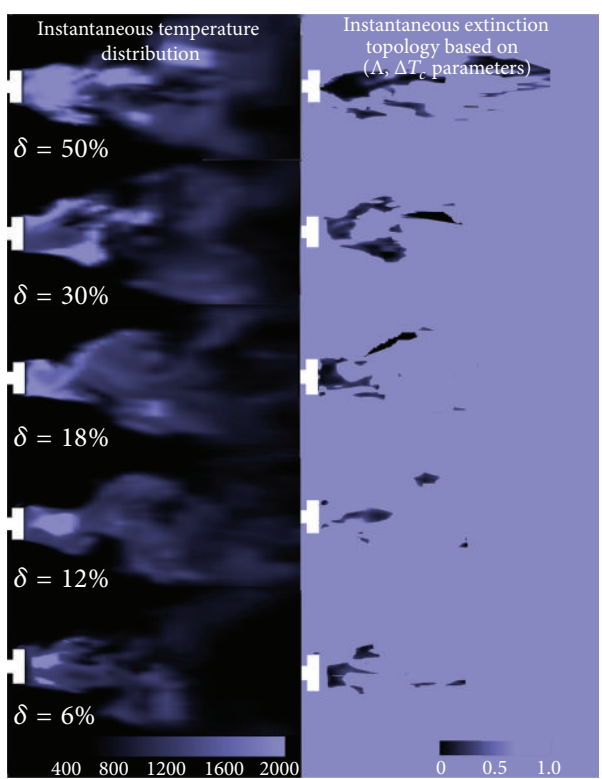

(a)

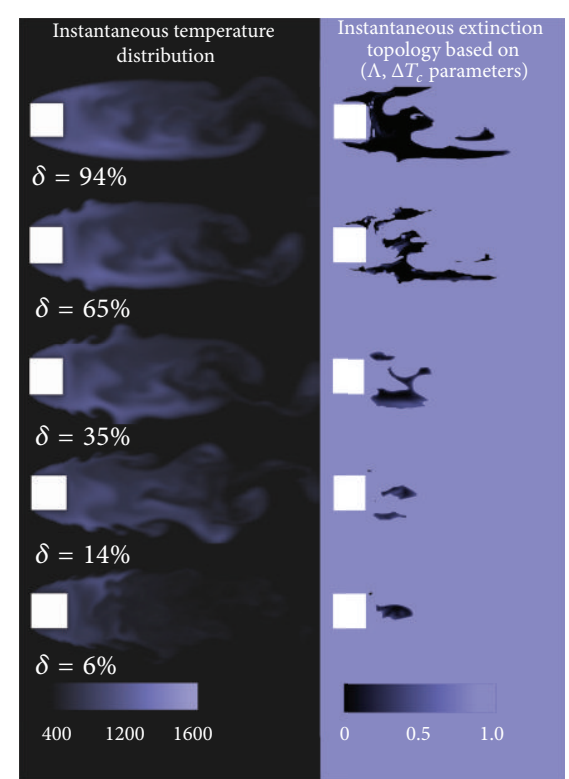

(b)

FIGURE 7: Snapshots of temperature and extinction topologies for a range of simulated flame settings as LBO is approached, (a) disk and (b) square burner.

The cross-stream traverses in Figure 6(b) (obtained at $\left.x / D_{b}=0.4\right)$ are consistent with the overall distributions shown in Figure 2(a) and suggest that a wider flame spread is obtained in the computed profiles. The peak $T$, located in line with the disk rim vortex where flame stabilization occurs, suffers a gradual drop from $1860 \mathrm{~K}$ to $1700 \mathrm{~K}$ and then to $1500 \mathrm{~K}$ with the leaner profile producing a more discernible off-axis temperature peak in accordance with the overall topology of Figure 2(a). These distributions could offer some guidance as far as the possible methodologies, for example, pilot injection placement that can be adopted to mitigate the evolution toward LBO in the stabilization region close to the burner rim.

The influence of varying the local equivalence ratio stratification on the simulated and measured time-mean centerplane and cross-stream (taken at $x / h=0.6$ ) temperature distributions for the square burner and for the fuel levels already seen in Figure 3(a) is depicted in the plots of Figures 6(c) and 6(d). Significantly lower temperatures, within a much longer recirculation zone, are now exhibited in this topology, and this trend is maintained for all successive fuel reductions. Peak values are found at the flanks of the developing wake of the square center-body, at $y / h=0.9$, in line with a more spreadout flame front stabilization disposition due to the displacing effect of the flanking recirculations on the square sides (Figures 3(a) and 3(b)). In fully premixed slender bluff-body stabilization setups studied in, for example, $[6,12]$, the flame was distinctly drawn within the recirculation and interacted with the reappearing vortex shedding mechanism. Here the adjacent flame fronts, with no apparent mutual interaction at the lean and ultra-lean level, gradually retract along the flanks of the wake engulfing the hot product vortex zone and it can be conjectured that this separation effectively delays the full reemergence of the Von Karman instability out of the formation region [11, 19]. These characteristics will be further verified from instantaneous images presented below.

Figures 7(a) and 7(b) display a sequence of LES snapshots of the temperature and the corresponding extinction topology as inferred from two of the criteria discussed previously for a range of fuel settings as we get closer to LBO. The dark areas demarcate reactive regions where both the Karlovitz $(\Lambda)$ and the temperature threshold $\left(\Delta T_{c}\right)$ criteria are simultaneously valid. Within the present formalism, this is a necessary but not a sufficient condition for sustaining reaction within the indicated areas. From these plots, it appears (Figure 7(a)) that once the side rim vortex loses its stabilizing effectiveness, the flame destabilizes and is unable to recover despite the occasional excursion of reacting fragments into the core of the main recirculation.

The square extinction topology (Figure 7(b)) indicates an efficient and resistant stabilization of the two reacting fronts within the flanking side vortices. However, it appears that when the two reactive layers are sufficiently retracted, the inherent intense large-scale instability $[12,17]$ is reinstated at the flame trailing edges. This has a detrimental effect on the overall stability, and the attribute of the anchoring in the strong flanking vortices is cancelled, leading thereafter to a more rapid approach to LBO in the final stages with respect to the disk behavior. Animation sequences from the simulations, supplied as supplementary material and taken after a sudden fuel reduction to the LBO levels, provide a more detailed illustration of the flow and flame events that occur at the final stages of the blow-out process for the two burners.

The local parameters used to monitor the local extinction behavior are discussed next. The instantaneous scatterplots of the simulated Karlovitz parameter monitored at a position close to the trailing edge (corner) of the two stabilizers, next to the temperature shear layer(s) (at $x / D_{b}, r / D_{b}=0.32,0.4$ 


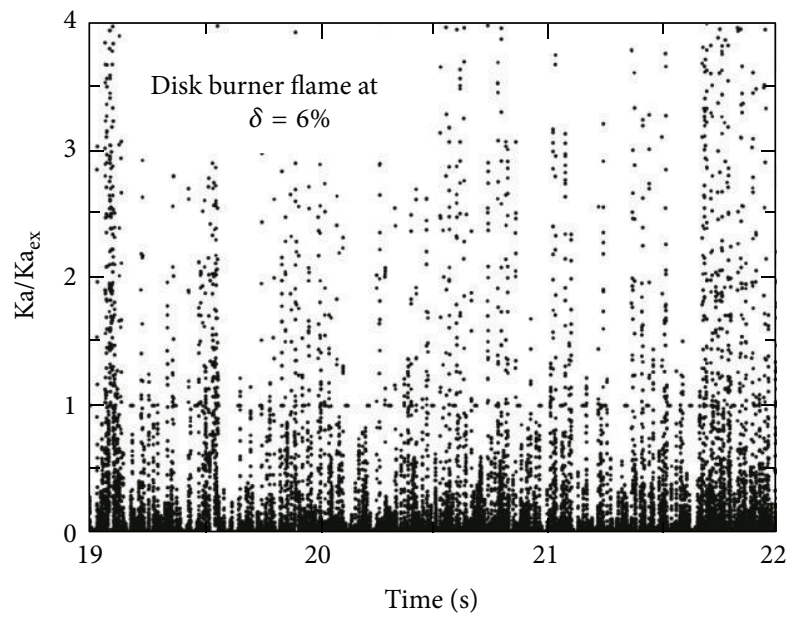

(a)

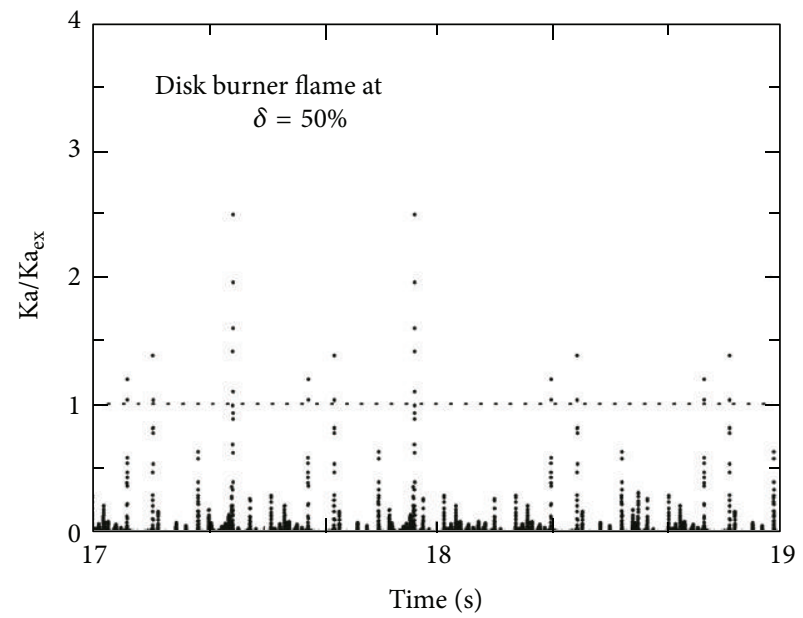

(b)

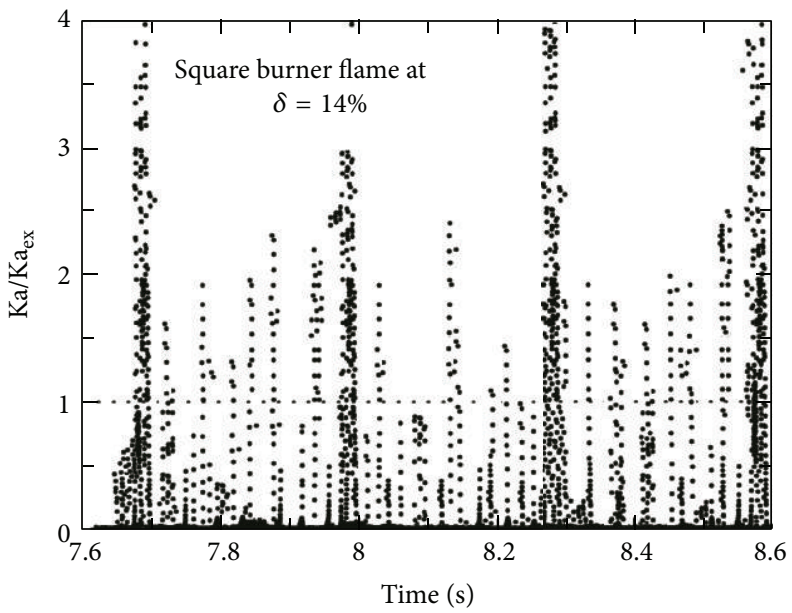

(c)

Figure 8: Scatterplots of the normalized Karlovitz number for the disk ((a), (b)) and the square (c) at different levels of local extinction $(\Lambda=$ $\mathrm{Ka} / \mathrm{Ka}_{\mathrm{ex}}$ values limited to 4 for clarity of extinction events signified by values greater than 1 ).

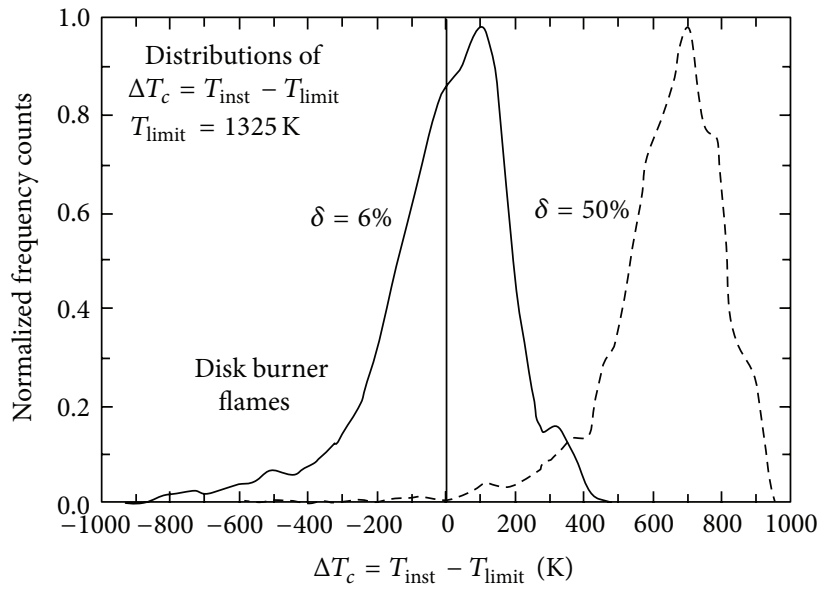

(a)

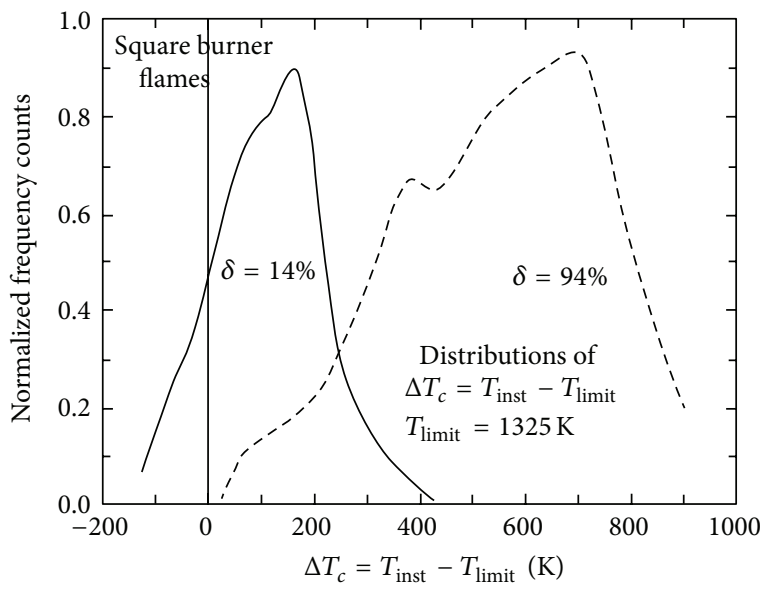

(b)

FIGURE 9: Statistical distributions of the instantaneous temperature difference parameter for the disk (a) and the square (b) at different levels of local extinction. 


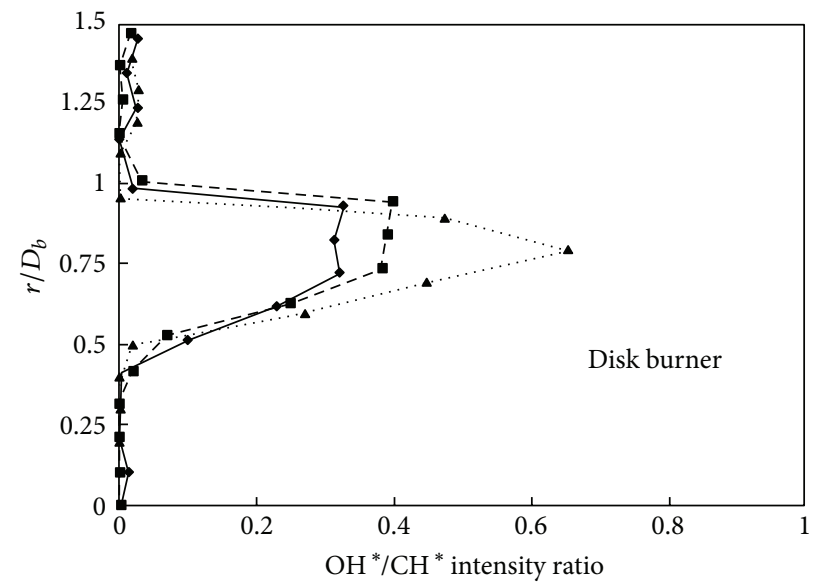

$\begin{aligned} \longrightarrow & =50 \% \\ --\delta & =18 \% \\ \cdots & =6 \%\end{aligned}$

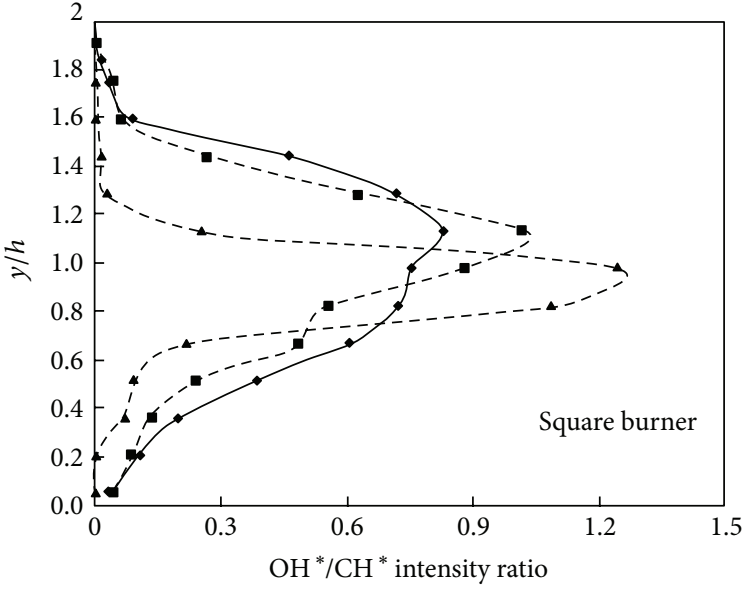

$\begin{aligned} \longrightarrow & \delta=94 \% \\ -\square & \delta=65 \% \\ -\bullet & \delta=14 \%\end{aligned}$

(a)

(b)

FIGURE 10: Cross-stream profiles of the ratio of the mean $\mathrm{OH}^{*}$ to $\mathrm{CH}^{*}$ chemiluminescence across the flame front within the recirculation zone for three ultra-lean and limiting flame settings, (a) disk and (b) square burner. Chemiluminescence profiles were taken at $x / D_{b}=0.44$ for the disk and at $x / h=1.6$ for the square.

for the disk and at $x / h, y / h=0.5,0.7$ for the square), are shown in Figures $8(\mathrm{a}), 8(\mathrm{~b})$, and $8(\mathrm{c})$ for each burner and two levels of fuel reduction. Depending on the level of local extinction, these plots exhibit distinctly different dispersions consistent with the reduction of the local reactivity as determined by the Karlovitz criterion. Interestingly, the scatterplot distribution for the square, depicted in Figure 8(c), displays a recursive appearance of four major extinction events. This corroborates the onset of vortical development at the flame tongues that seem to be reinstated as LBO is approached, as also implied by the low $\delta$ snapshots of Figure 7(b). Such a behavior has been reported previously for fully premixed, for example, $[6,12]$ and partially premixed configurations [11].

The computed statistical distributions (frequency counts) of the instantaneous temperature threshold parameter, $\Delta T_{c}$, are displayed for the same monitoring position in Figures 9(a) and 9(b) for the two setups, respectively. A threshold temperature has been previously exploited as a practical and complementary diagnostic parameter for indicating extinction $[2,3,10]$. Based on the present experimental and computational tests and various studies on laminar counterflow [2, 10 ] and turbulent flames [3], a limit value of $1325 \mathrm{~K}$ was here tentatively used for the present conditions. The disposition of the successive distributions of $\Delta T_{c}$, shown in Figures 9(a) and 9 (b), consistently delineates the increased probability for local extinction; since the selected monitoring point lays close to the stabilizing shear layers, these, in combination with further monitoring parameters, could also be considered as indicative of the proximity to global extinction.

Figures 10(a) and 10(b) display the cross-stream profiles of the ratio of the time-mean $\mathrm{OH}^{*} / \mathrm{CH}^{*}$ chemiluminescence, within the recirculation regions and across the flame fronts, at $x / D_{b}=0.44$ for the disk and at $x / h=1.6$ for the square, away from the burner face. The $\mathrm{OH}^{*}$ and $\mathrm{CH}^{*}$ disk profiles have been projected onto an axisymmetric plane through an inverse Abel transform for a more consistent comparison of their ratio. The results imply that the flames closer to extinction consistently attain a higher value of this ratio for both burners, and this trend, if further elaborated, could likely be suitable for demarcating limiting conditions. It could be postulated that apart from monitoring the transient behavior of the above or additional parameters, at judiciously chosen locations, the examination of time-mean data could be equally useful in producing useful overall criteria, in a fashion similar to the exploitation of a local Damköhler number, an attribute that merits further verification over a range of flame topologies and conditions.

Figure 11 displays instantaneous $\mathrm{OH}^{*}$ chemiluminescence images obtained at a fuel setting of $\delta=6 \%$ for the disk and of $\delta=14 \%$ for the square, respectively. The axisymmetric reacting wake structures in the disk images indicate a flame topology with highly variable peripheral liftoff distance with time. These flame front structures form a wide angle relative to the incoming flow due to the effect of the high swirl intensity and seem to occasionally penetrate from the periphery toward the base of the flame within the recirculation region. The overall disposition of the flame front under stratification seems to differ both in terms of length, placement, and anchoring behaviour from both nonpremixed $[16,19]$ and fully premixed axisymmetric $[4,7,8]$ counterpart topologies. The transient $\mathrm{OH}^{*}$ chemiluminescent images for the square verify the two-tongued flame front placement previously implied by the time-mean experimental and simulation results (Figure 3 ). The two fronts independently reciprocate back and forth in time without any apparent interaction. The images do not provide any indication of 

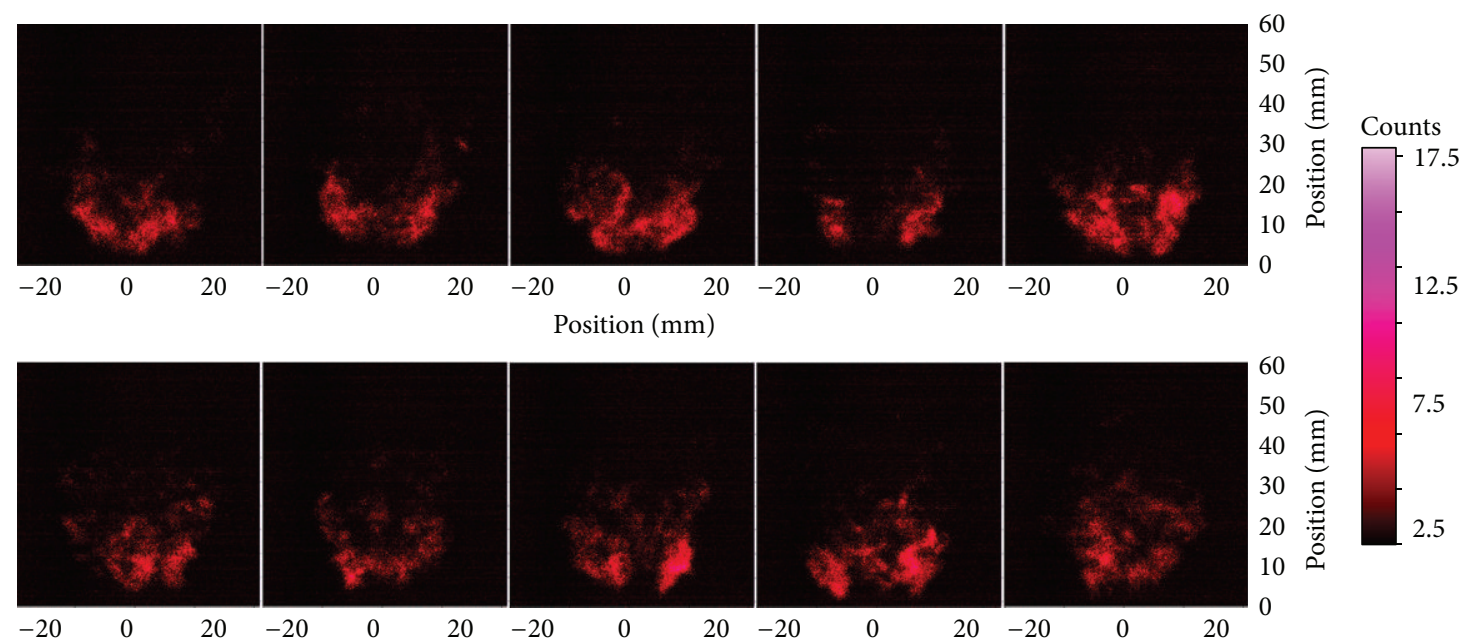

(a)
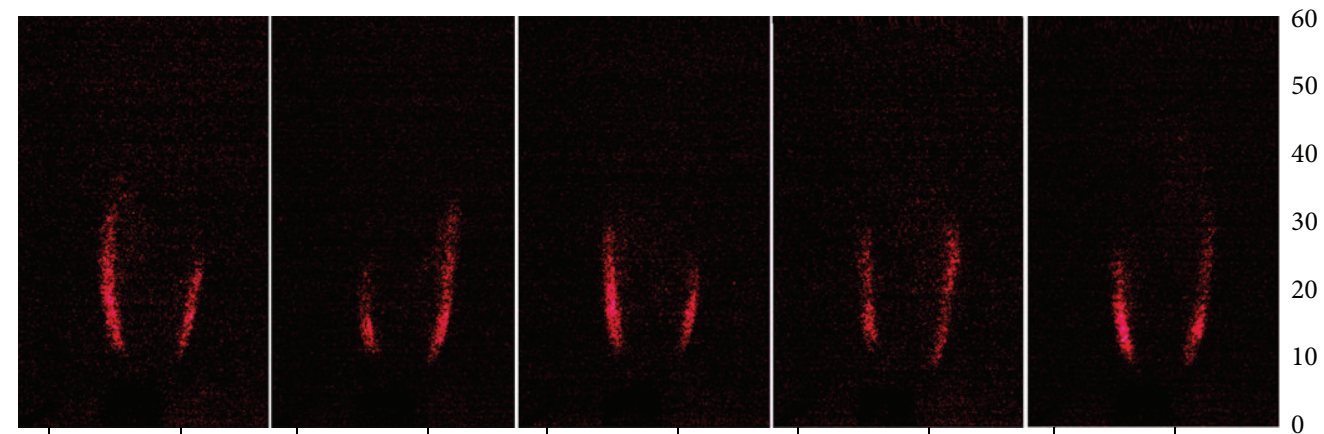

50

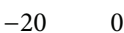

$20-20$

$20-20$

Position (mm)

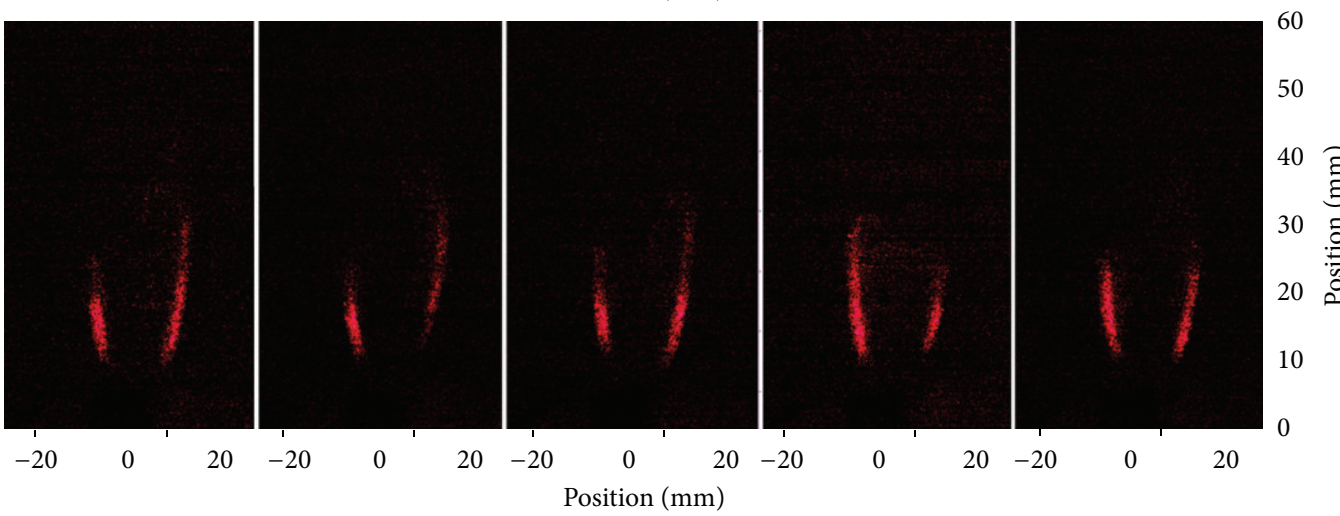

(b)

Figure 11: Instantaneous $\mathrm{OH}^{*}$ images for disk (a) and square (b) burner operating with a mixture level of $\delta=6 \%$ (disk) and $\delta=14 \%$ (square).

reaction within the formation region, which is in stark contrast to the reported behavior $[6,12]$ of ultralean and limiting slender body stabilization of fully premixed flames.

\section{Summary and Conclusions}

Measurements and simulations of axisymmetric swirling disk and $2 \mathrm{D}$ slender square bluff-body reacting wakes, working with an inlet mixture gradient, have been performed under ultra-lean and near blowoff conditions. Results have been presented and compared to highlight some differences and similarities in the flame characteristics of each stabilizer under a variable stratified inlet mixture profile sustained across the leading edge flame front stabilization region. These data can also provide a useful assessment of the ultra-lean operation of these two popular stabilizers under stratified 
inlet mixture conditions as compared against the more traditional fully premixed operation.

The axisymmetric flames exhibited a more compact, frustum-like shaped near fame region, with significant radial spread dependent on swirl intensity. Recirculating hot products occupied a toroidal region with an extent of about 20 to $40 \%$ longer than the isothermal length. This was maintained across the examined mixture strengths and was shorter than that found in counterpart fully premixed axisymmetric cases. Over the ultra-lean range and at the limiting flame conditions reacting, front fragments were identified to penetrate within this recirculation region from the rim vortex stabilization region. The formation of ring vortical structures that has been identified in toroidal disk flames at fully premixed operation was somewhat curtailed under stratified operation, and this aspect requires further investigations since it affects the stability of these flames.

The slender square body flames were stabilized at the rim vortices formed on the square flanks, establishing a twotongued appearance. The lengths of the two individual reacting fronts were from two to eight square heights depending on mixture strength. Similarly, with reaction, the cold wake formation region was expanded by about four to five times in the lean cases; this was drastically shortened at the weaker inlet mixtures. Peak temperatures for the disk were located both in the toroidal shear layer and extended well within the recirculation zone, while for the square these were clearly positioned along the square flanks. The development of VonKarman vortical instability was clearly identified to emerge in the square flames as the flame fronts were significantly withdrawn back closer to the burner face; this behaviour is also different from fully premixed configurations that have been reported to maintain periodic symmetric or antisymmetric vortical structures across a wide range of equivalence ratios.

The adopted LES procedure with an appropriate choice of combustion and chemistry submodels reproduced satisfactorily the measured mean velocity and temperature distributions while providing useful qualitative descriptions of the examined species fields which are compared quite favourably with the chemiluminescence imaging measurements. The method also provided some insight into the topology of the leading edge flame front anchoring adjacent to the flanks of axisymmetric or plane geometry stabilizers, in the vicinity of equivalent ratio peaks, within the context of a stratified inlet mixture gradient for ultra-lean and near to blowoff conditions.

\section{Conflict of Interests}

There is no conflict of interests that arises from the methodologies, the instrumentations, or the software used for the production and appraisal of the present research results within the context of the present work performed by the authors.

\section{Acknowledgment}

This work was partly supported by the Research Council of the University of Patras.

\section{References}

[1] B. W. Bilger, S. B. Pope, K. N. C. Bray, and J. F. Driscoll, "Paradigms in turbulent combustion research," Proceedings of the Combustion Institute, vol. 30, no. 1, pp. 21-42, 2005.

[2] D. Bradley, "Combustion and the design of future engine fuels," Proceedings of the Institution of Mechanical Engineers C, vol. 223, no. 12, pp. 2751-2765, 2009.

[3] G. E. Andrews, N. T. Ahmed, R. Phylaktou, and P. King, "Weak extinction in low NOx gas turbine combustion," in Proceedings of the ASME Turbo Expo, pp. 623-638, June 2009.

[4] M. Stohr, I. Boxx, C. Carter et al., "Dynamics of lean blowout of a swirl-stabilized flame in a gas turbine model combustor," Proceedings of the Combustion Institute, vol. 33, no. 2, pp. $2953-$ 2960, 2011.

[5] M. S. Sweeney, S. Hochgreb, and R. S. Barlow, "The structure of premixed and stratified low turbulence flames," Combustion and Flame, vol. 158, no. 5, pp. 935-948, 2011.

[6] S. G. Tuttle, S. Chaudhuri, K. M. Kopp-Vaughan et al., "Blowoff dynamics of asymmetrically-fueled, bluffbody flames," in Proceedings of the 49th AIAA Aerospace Sciences Meeting, AIAA, Orlando, Fla, USA, 2011.

[7] J. R. Dawson, R. L. Gordon, J. Kariuki et al., "Visualization of blow-off events in bluff-body stabilized turbulent premixed flames," Proceedings of the Combustion Institute, vol. 33, no. 1, pp. 1559-1566, 2011.

[8] Q. Zhang, S. J. Shanbhogue, T. Lieuwen, and J. O'Connor, “Strain characteristics near the flame attachment point in a swirling flow," Combustion Science and Technology, vol. 183, no. 7, pp. 665-685, 2011.

[9] C. Duwig, K.-J. Nogenmyr, C.-K. Chan, and M. J. Dunn, "Large eddy simulations of a piloted lean premix jet flame using finiterate chemistry," Combustion Theory and Modelling, vol. 15, no. 4, pp. 537-568, 2011.

[10] E.-S. Cho, S. H. Chung, and T. K. Oh, "Local karlovitz numbers at extinction for various fuels in counterflow premixed flames," Combustion Science and Technology, vol. 178, no. 9, pp. 1559$1584,2006$.

[11] P. Koutmos and K. Souflas, "A study of slender bluff body reacting wakes formed by con-current or counter-current fuel injection," Combustion Science and Technology, vol. 184, no. 9, pp. 1343-1365, 2012.

[12] K. M. Kopp-Vaughan, T. R. Jensen, B. M. Cetegen et al., "Analysis of blowoff dynamics from flames with stratified fueling," Proceedings of the Combustion Institute, vol. 34, no. 1, pp. 14911498, 2013.

[13] C. Z. Xiouris and P. Koutmos, "Fluid dynamics modeling of a stratified disk burner in swirl co-flow," Applied Thermal Engineering, vol. 35, no. 1, pp. 60-70, 2012.

[14] O. Colin, F. Ducros, D. Veynante, and T. Poinsot, "A thickened flame model for large eddy simulations of turbulent premixed combustion," Physics of Fluids, vol. 12, no. 7, pp. 1843-1863, 2000.

[15] G. Wang, M. Boileau, and D. Veynante, "Implementation of a dynamic thickened flame model for large eddy simulations of turbulent premixed combustion," Combustion and Flame, vol. 158, no. 11, pp. 2199-2213, 2011.

[16] P. Koutmos, G. Paterakis, E. Dogkas, and C. H. Karagiannaki, "The impact of variable inlet mixture stratification on flame topology and emissions performance of a premixer/swirl burner configuration," Journal of Combustion, vol. 2012, Article ID 374089, 12 pages, 2012. 
[17] R. R. Erickson and M. C. Soteriou, "The influence of reactant temperature on the dynamics of bluff body stabilized premixed flames," Combustion and Flame, vol. 158, no. 12, pp. 2441-2457, 2011.

[18] J. J. Miau, T. S. Leu, T. W. Liu, and J. H. Chou, "On vortex shedding behind a circular disk," Experiments in Fluids, vol. 23, no. 3, pp. 225-233, 1997.

[19] A. G. Bakrozis, D. D. Papailiou, and P. Koutmos, "A study of the turbulent structure of a two-dimensional diffusion flame formed behind a slender bluff-body," Combustion and Flame, vol. 119, no. 3, pp. 291-306, 1999.

[20] D. Trimis, Verbrennungsvorgange in Porosen Inerten Medien, BEV 95.5, ESYTEC, Elangen, Germany, 1996.

[21] C. Dasch, "One-dimensional tomography: a comparison of Abel, onion-peeling, and filtered back-projection methods," Applied Optics, vol. 31, no. 8, pp. 1146-1152, 1992.

[22] ANSYS Fluent, Release 12.0, Theory Guide, ANSYS, 2009.

[23] L. Gicquel, G. Staffelbach, and T. Poinsot, "Large Eddy Simulations of gaseous flames in gas turbine combustion chambers," Progress in Energy and Combustion Science, vol. 38, no. 6, pp. 782-817, 2012.

[24] A. Briones and B. Sekar, Effect of Von Kármán Vortex Shedding on Regular and Open-Slit V-Gutter Stabilized Turbulent Premixed Flames, Spring Technical Meeting of the Central States Section of the Combustion Institute, University of Dayton, Air Force Research Laboratory Dayton, 2012. 

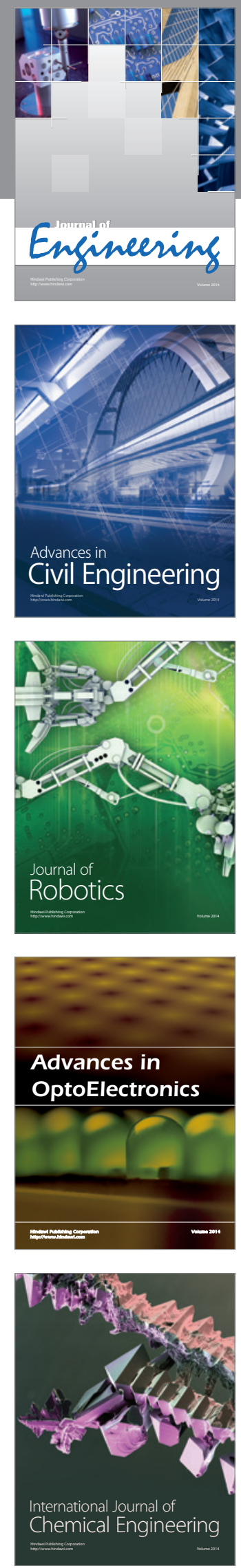

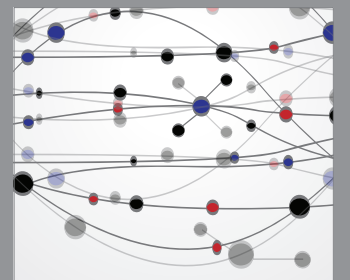

The Scientific World Journal
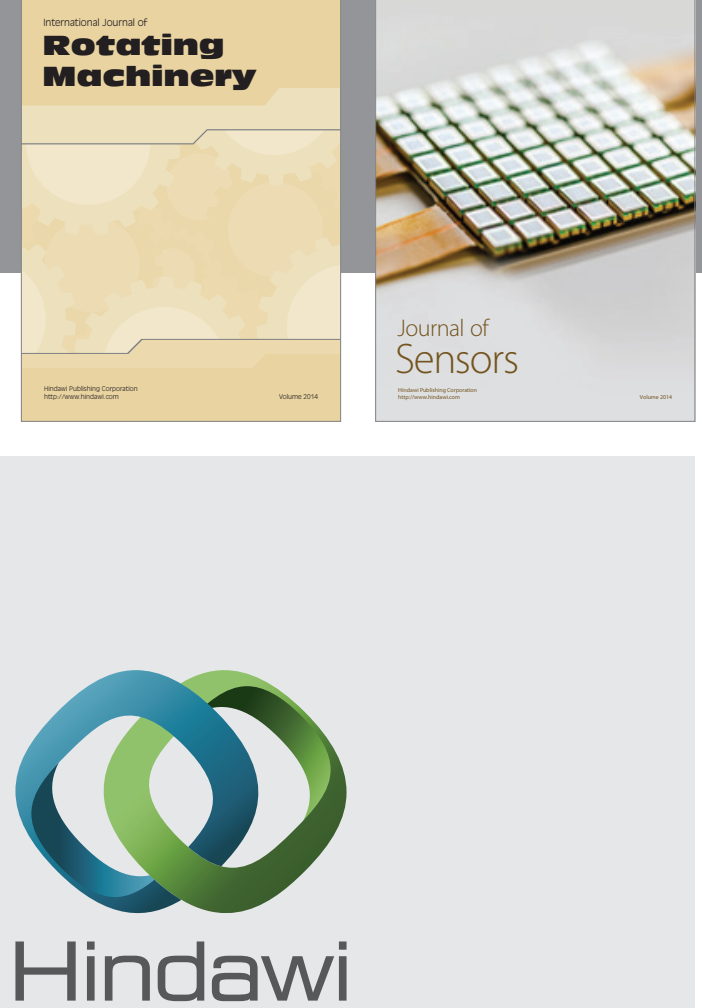

Submit your manuscripts at http://www.hindawi.com
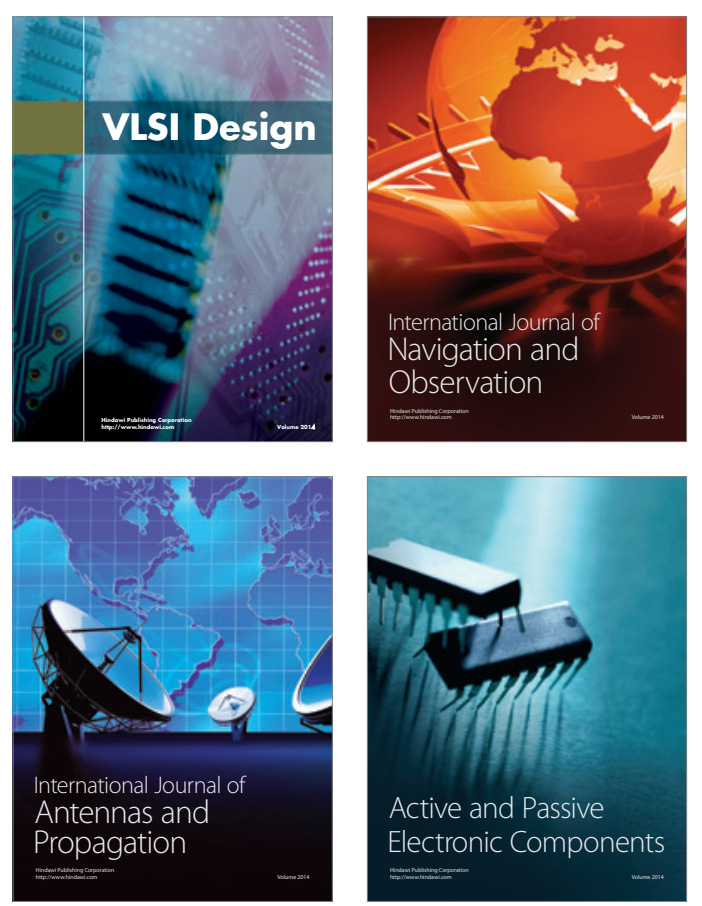
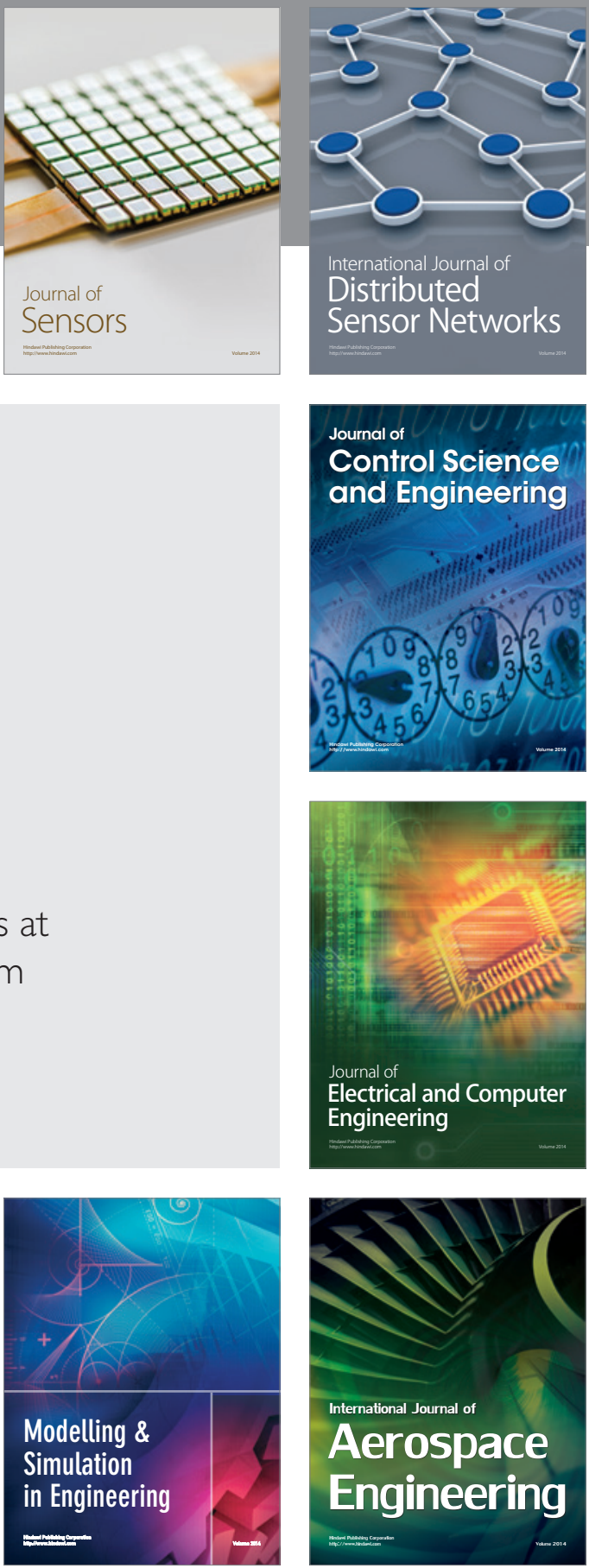

Journal of

Control Science

and Engineering
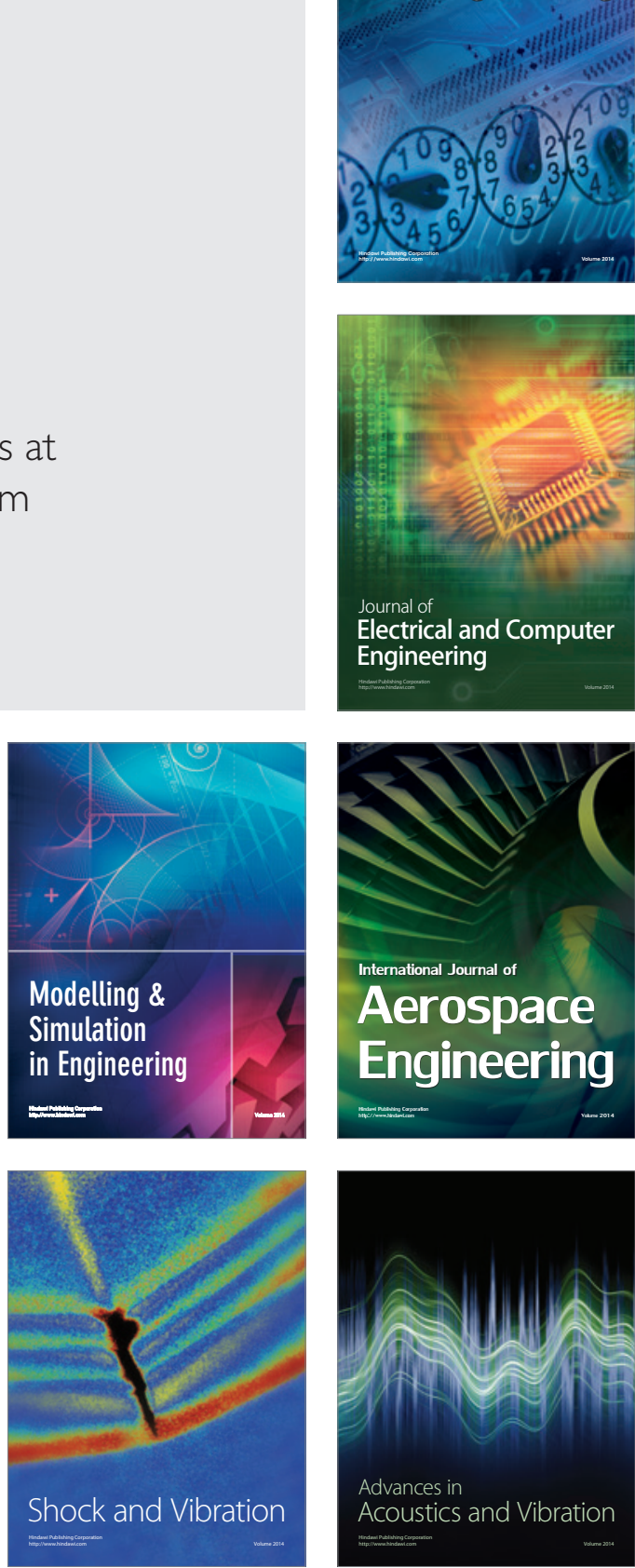